2013-03-20

\title{
Passive Stiffness of Coupled Wrist and Forearm Rotations
}

Will Brandon Drake

Brigham Young University - Provo

Follow this and additional works at: https://scholarsarchive.byu.edu/etd

Part of the Mechanical Engineering Commons

\section{BYU ScholarsArchive Citation}

Drake, Will Brandon, "Passive Stiffness of Coupled Wrist and Forearm Rotations" (2013). Theses and Dissertations. 3446.

https://scholarsarchive.byu.edu/etd/3446

This Thesis is brought to you for free and open access by BYU ScholarsArchive. It has been accepted for inclusion in Theses and Dissertations by an authorized administrator of BYU ScholarsArchive. For more information, please contact scholarsarchive@byu.edu, ellen_amatangelo@byu.edu. 
Passive Stiffness of Coupled Wrist and Forearm Rotations

Will Brandon Drake

A thesis submitted to the faculty of

Brigham Young University

in partial fulfillment of the requirements for the degree of

Master of Science

Steven K. Charles, Chair

Anton E. Bowden

Larry L. Howell

Department of Mechanical Engineering

Brigham Young University

March 2013

Copyright (C) 2013 Will Brandon Drake

All Rights Reserved 


\author{
ABSTRACT \\ Passive Stiffness of Coupled Wrist and Forearm Rotations \\ Will Brandon Drake \\ Department of Mechanical Engineering, BYU \\ Master of Science
}

The dynamics of wrist rotations are dominated by joint stiffness, which the neuromuscular system must account and compensate for when controlling wrist movements. Because wrist stiffness is anisotropic, movements in some directions require less torque than movements in others, creating opportunities to follow "paths of least resistance." Forearm pronation-supination (PS) can combine with wrist flexion-extension (FE) and radial-ulnar deviation (RUD) to allow the wrist to rotate in directions of least stiffness. Evaluating this hypothesis, and understanding the control of combined wrist and forearm rotations in general, requires a knowledge of the stiffness (the dominant mechanical impedance) encountered during combined wrist and forearm rotations. While wrist and forearm stiffness have been measured in isolation, there are no measurements of coupled wrist and the forearm stiffness. This study characterizes the passive stiffness of the wrist and forearm in combinations of FE, RUD, and PS. Using a wrist and forearm robot, we measured coupled wrist and forearm stiffness for $15^{\circ}$ movements from neutral position in 10 young, healthy subjects. We found the stiffness in PS to be significantly smaller than the stiffness in RUD, but similar in magnitude to the stiffness in FE, indicating that the torque required to overcome stiffness in combinations of PS and FE is significantly smaller than the torque required to overcome stiffness in combinations of FE and RUD (assuming equal displacements). The coupled stiffness measured here will enable future studies to determine optimal paths and to compare these optimal paths to observed movements involving wrist and forearm rotations.

Keywords: wrist, forearm, stiffness, passive, muscle tone, motor control 


\section{ACKNOWLEDGMENTS}

The author would like to express his immense appreciation for Dr. Steven K. Charles, under whose tutelage the author has grown considerably as an engineer, a researcher, and as a person, and without whom this research and thesis would never have become a reality. The author would also like to express his deep gratitude and love for his wife Johannah, whose patience and constant love have been a never-failing source of strength during these past two years. 


\section{TABLE OF CONTENTS}

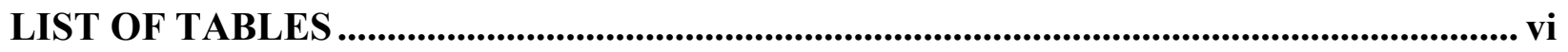

LIST OF FIGURES ..........................................................................................................

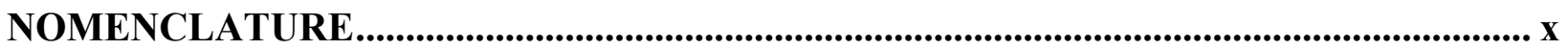

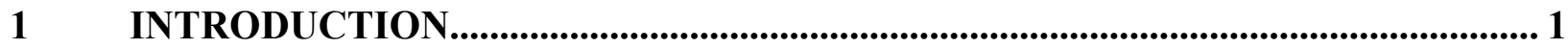

2 LITERATURE REVIEW ........................................................................................ 5

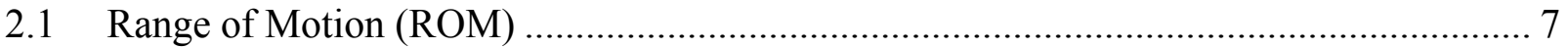

2.2 Definition of Neutral Position and Arm Orientation ..................................................... 13

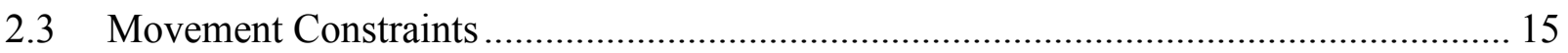

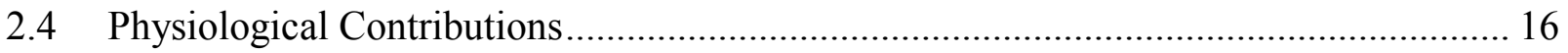

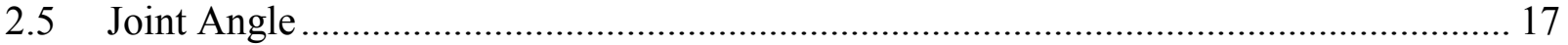

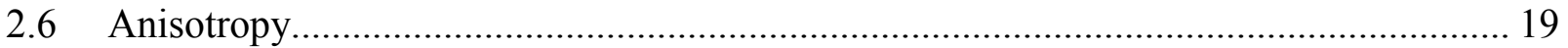

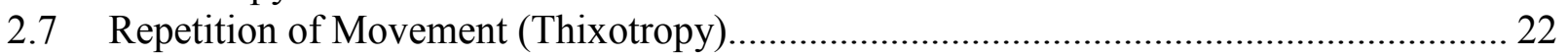

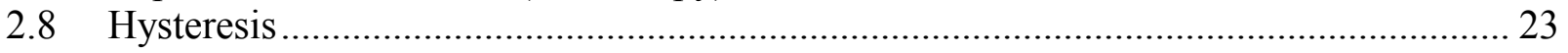

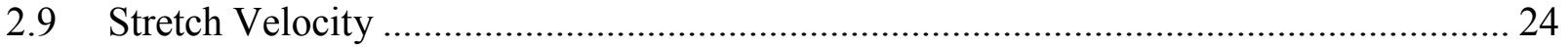

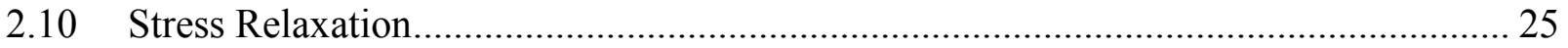

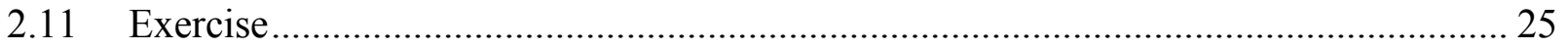

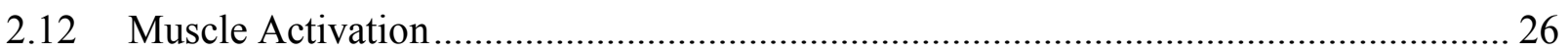

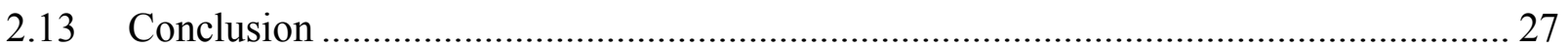

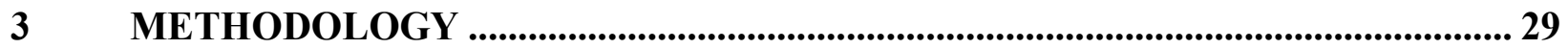

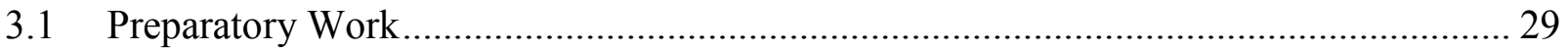

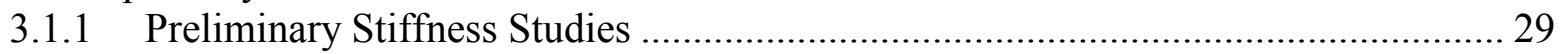

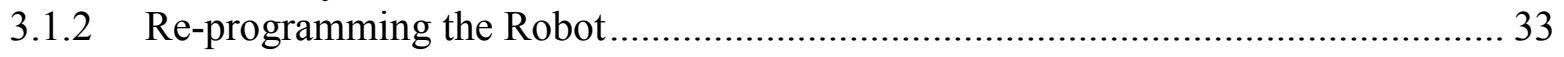

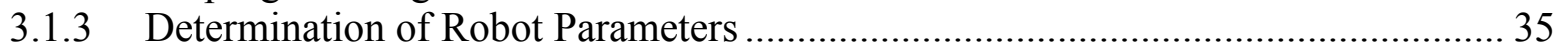

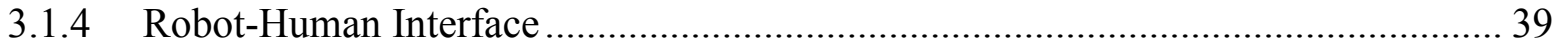

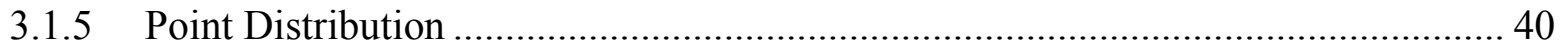

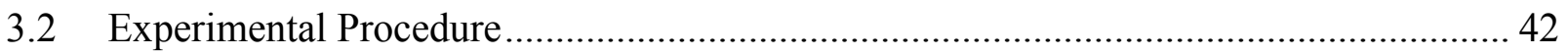

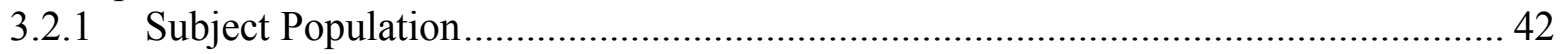

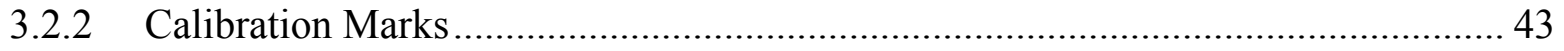

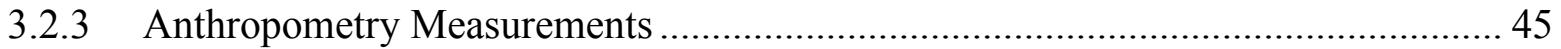

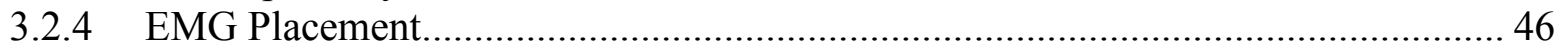

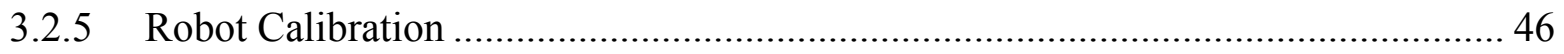

3.2.6 Maximum Voluntary Contraction ....................................................................... 48

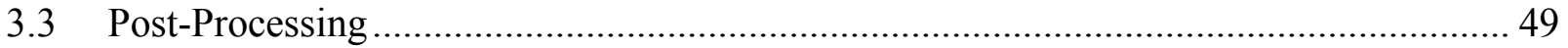

3.3.1 Torque-Position Processing ............................................................................. 49

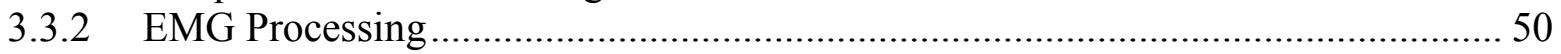

3.3.3 Robot Dynamics and Gravity Compensation .................................................... 50

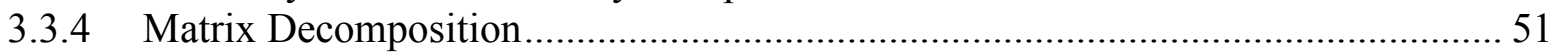

4 CENTRAL EXPERIMENT AND RESULTS ............................................................5 53

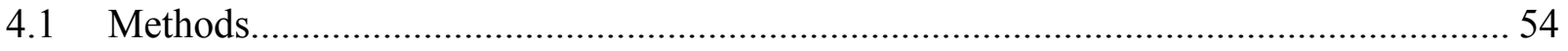

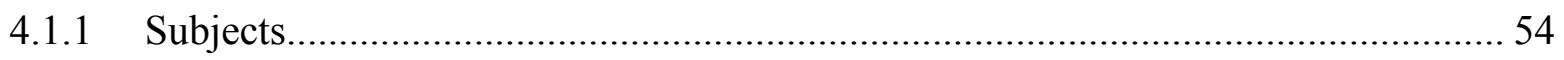




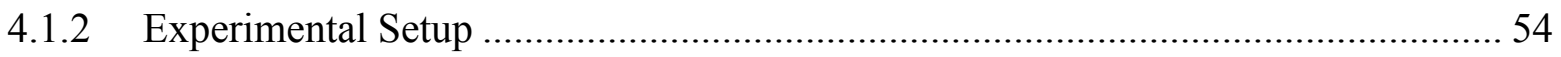

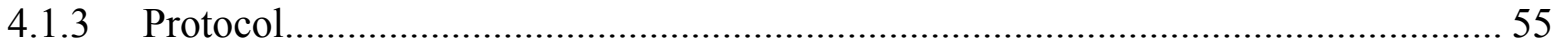

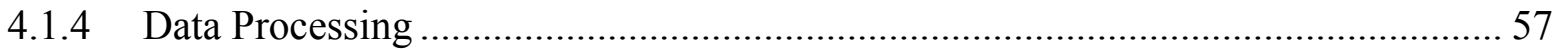

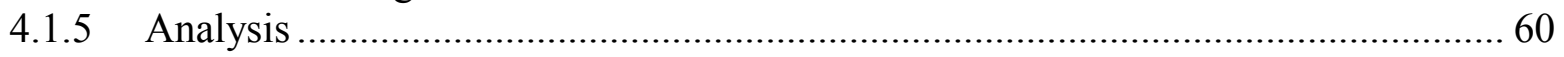

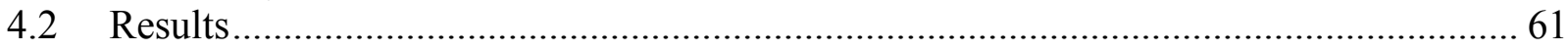

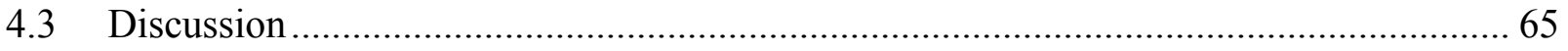

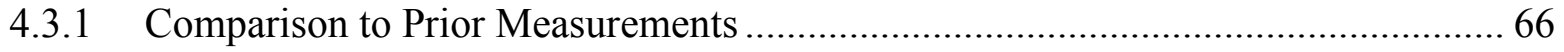

4.3.2 Implications for the Neural Control of Movement............................................. 69

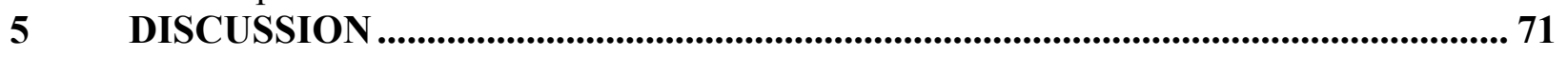

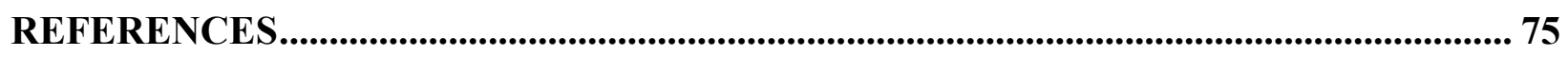




\section{LIST OF TABLES}

Table 2-1: Selected results from extreme range-of-motion studies. ...................................... 9

Table 2-2: Results from functional range-of-motion wrist studies......................................... 12

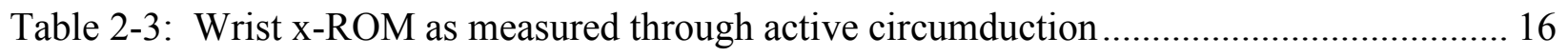

Table 2-4: Reported values of passive wrist stiffness.................................................... 21

Table 4-1: Mean compensated stiffness matrices and standard deviations. ............................ 62

Table 4-2: Comparison of ellipsoid shape, orientation, and volume ....................................... 63

Table 5-1: Comparison of our results to prior measurements of wrist stiffness........................ 72 


\section{LIST OF FIGURES}

Figure 1-1: Definitions of wrist and forearm movement ............................................... 3

Figure 2-1: Stereotypical plot of wrist ROM throughout circumduction. ................................ 13

Figure 2-2: Torque response for passive movement of the metacarpophalangeal joint. ............ 18

Figure 2-3: Torque response for passive extension and flexion of the wrist joint..................... 19

Figure 2-4: Torque responses to passive wrist extensions exceeding the SRS limit................ 23

Figure 3-1: Comparison of passive wrist stiffness measurements ......................................... 34

Figure 3-2: Comparison of torque-position to determine operating parameters......................... 36

Figure 3-3: Torque-position plots for a typical experimental run........................................ 38

Figure 3-4: Average of R2-R5 for the velocity test presented in Figure 3-3 ........................... 38

Figure 3-5: Midrange stiffness estimate in flexion for varying test velocities. ......................... 39

Figure 3-6: Custom-built robot-hand interface. ............................................................... 40

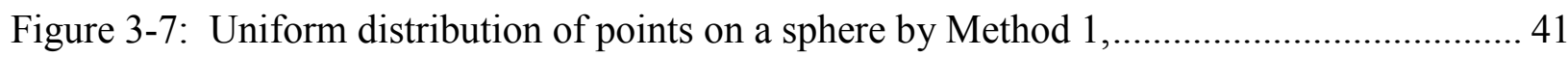

Figure 3-8: Uniform distribution of points on a sphere by Method 2 .................................... 42

Figure 3-9: Anthropometric data sheet used in the study. .................................................... 47

Figure 4-1: Plots of the point distribution used during the study. ......................................... 56

Figure 4-2: Progression of the torque-displacement data from raw data to processed data....... 61

Figure 4-3: Stiffness matrices displayed as stiffness ellipsoids.......................................... 63

Figure 4-4: Mean and standard deviation of eigenvectors for all stiffness ellipsoids. .............. 64

Figure 4-5: Mean, standard error, and range of the volume of stiffness ellipsoids .................. 65

Figure 4-6: Comparison of our results to prior measurements of wrist stiffness..................... 68 


\section{NOMENCLATURE}

\begin{tabular}{|c|c|}
\hline$A D L$ & Activities of daily living \\
\hline$A N O V A$ & Analysis of variance \\
\hline$B I C$ & Biceps brachii \\
\hline$D A C 3$ & The distal tip of the third dactilion \\
\hline$D E M$ & $\begin{array}{l}\text { Dorsal edge mark. Mark made at the proximal edge of the dynamometer on dorsal } \\
\text { side of the subject's hand. }\end{array}$ \\
\hline$E C R$ & Muscle group comprised of the extensor carpi radialis brevis and longus muscles \\
\hline$E C U$ & Extensor carpi ulnaris \\
\hline EJC & Elbow joint center \\
\hline$E M G$ & Electromyography \\
\hline$E T$ & Elbow trough mark \\
\hline$F C R$ & Flexor carpi radialis \\
\hline$F C U$ & Flexor carpi ulnaris \\
\hline$F E$ & Flexion-extension of the wrist joint \\
\hline$f-R O M$ & Functional range of motion as measured during $A D L$ \\
\hline$K$ & The $3 \times 3$ passive stiffness matrix \\
\hline$K_{F E, F E}$ & An element of the stiffness matrix $K$. The change in $\tau_{F E}$ that occurs with $\theta_{F E}$. \\
\hline$K_{F E, P S}$ & $\begin{array}{l}\text { An element of the stiffness matrix } K \text {. The change in } \tau_{F E} \text { that occurs with } \theta_{P S} \text {. } \\
\text { Equivalent to } K_{P S, F E} \text { after discarding the anti-symmetric portion of } K \text {. }\end{array}$ \\
\hline$K_{F E, R U D}$ & $\begin{array}{l}\text { An element of the stiffness matrix } K \text {. The change in } \tau_{F E} \text { that occurs with } \theta_{R U D} \text {. } \\
\text { Equivalent to } K_{R U D, F E} \text { after discarding the anti-symmetric portion of } K \text {. }\end{array}$ \\
\hline$K_{P S, P S}$ & An element of the stiffness matrix $K$. The change in $\tau_{P S}$ that occurs with $\theta_{P S}$. \\
\hline$K_{P S, F E}$ & $\begin{array}{l}\text { An element of the stiffness matrix } K \text {. The change in } \tau_{P S} \text { that occurs with } \theta_{F E} \text {. } \\
\text { Equivalent to } K_{F E, P S} \text { after discarding the anti-symmetric portion of } K \text {. }\end{array}$ \\
\hline$K_{P S, R U D}$ & $\begin{array}{l}\text { An element of the stiffness matrix } K \text {. The change in } \tau_{P S} \text { that occurs with } \theta_{R U D} \text {. } \\
\text { Equivalent to } K_{R U D, P S} \text { after discarding the anti-symmetric portion of } K \text {. }\end{array}$ \\
\hline$K_{R U D, F E}$ & $\begin{array}{l}\text { An element of the stiffness matrix } K \text {. The change in } \tau_{R U D} \text { that occurs with } \theta_{F E} \text {. } \\
\text { Equivalent to } K_{F E, R U D} \text { after discarding the anti-symmetric portion of } K \text {. }\end{array}$ \\
\hline$K_{R U D, P S}$ & $\begin{array}{l}\text { An element of the stiffness matrix } K \text {. The change in } \tau_{R U D} \text { that occurs with } \theta_{P S} \text {. } \\
\text { Equivalent to } K_{P S, R U D} \text { after discarding the anti-symmetric portion of } K \text {. }\end{array}$ \\
\hline$K_{R U D, R U D}$ & An element of the stiffness matrix $K$. The change in $\tau_{R U D}$ that occurs with $\theta_{R U D}$. \\
\hline$\lambda_{1}$ & The major eigenvalue of the symmetric portion of $K$. \\
\hline$\lambda_{2}$ & The intermediate eigenvalue of the symmetric portion of $K$. \\
\hline$\lambda_{3}$ & The minor eigenvalue of the symmetric portion of $K$. \\
\hline$L-W J C$ & WJC mark on the lateral face of the wrist joint \\
\hline$M C P 2$ & Metacarpophalangeal joint of the second digit. \\
\hline$M C P 3$ & Metacarpophalangeal joint of the third digit. \\
\hline$M R S$ & Midrange stiffness \\
\hline
\end{tabular}


MVC Maximum voluntary contraction reading for EMG

PEM Palmar edge mark. Mark made at the proximal edge of the dynamometer on subject's palm.

PRO Pronator teres

$P S \quad$ Pronation-supination of the forearm

ROM Range of motion

RUD Radioulnar deviation of the wrist joint

SRS Short range stiffness

$\tau_{F E}$

The torque experienced by the wrist joint during movements in FE

$\tau_{P S}$

The torque experienced by the forearm during movements in PS

$\tau_{R U D}$

The torque experienced by the wrist joint during movements in RUD

$\theta_{F E}$

Angular displacement of the wrist joint in FE

$\theta_{P S}$

Angular displacement of the forearm in PS

$\theta_{R U D}$

Angular displacement of the wrist joint in RUD

WJC Wrist joint center

$x$-ROM Extreme range of motion. The range measured during circumduction. 


\section{INTRODUCTION}

The wrist joint is essential in accomplishing activities of daily living (ADL. While the shoulder and elbow joints contribute greatly to the task of orienting the hand, the wrist joint directly carries the hand and makes the final contribution to place it in the optimal position to accomplish required tasks. The critical role that the wrist plays in accomplishing everyday activities is no more keenly felt than when the joint's performance is compromised either by injury or by chronic disorder. To give just one economic indicator of the importance of healthy wrist function, a six-year study of workers in Washington state found that workers suffering from carpal tunnel syndrome resulted in an average loss of earnings between $\$ 45,000$ and $\$ 89,000$ (Foley et al., 2007).

Improvements in ergonomic design, preventative medicine, surgical treatments, and wrist rehabilitation require an accurate characterization of healthy wrist movement and dynamics. In addition, coordinated motor control requires that the neuromuscular system account and compensate for joint dynamics. Deeper understanding of wrist joint dynamics will improve our models for describing the process used by the neuromuscular system to plan and coordinate movements involving the wrist.

While inertia has the greatest effect on dynamics for many joints, the torque required to overcome passive stiffness of the wrist joint during comfortably paced wrist rotations has been shown to be approximately ten times larger than the torque required to overcome the inertia of the hand (Charles and Hogan, 2011), making wrist stiffness the dominant effect. In fact, the 
observation of stereotyped curvature of active wrist movements can be explained by incorporating passive joint stiffness into dynamics models (Charles and Hogan, 2010, 2012). Because passive stiffness is the largest factor influencing wrist dynamics and movement, it needs to be thoroughly characterized: its factors identified, their effects quantified, their origin explained, and their combinatory effects modeled.

A survey of the literature reveals several key findings about passive wrist stiffness: 1) Wrist stiffness is anisotropic in nature, with greater stiffness in radial-ulnar deviation (RUD) than in flexion-extension (FE) (See Figure 1-1 for definition of wrist and forearm motion). 2) Wrist stiffness does not align with the anatomical axes of the wrist, coupling FE and RUD (i.e. a movement in pure FE induces a torque in RUD, and vice versa) (Charles and Hogan, 2011). 3) Wrist range-of-motion (ROM), a factor directly governed by passive stiffness, depends on finger position, decreasing by as much as $27 \%$ in flexion and by $11 \%$ in ulnar deviation depending on how the fingers are constrained-e.g. free, holding a handle, or constrained to a fist. 4) The wrist joint exhibits high stiffness over the initial $3^{\circ}-4^{\circ}$ of movement (short-range stiffness) (Axelson and Hagbarth, 2001). 5) The wrist joint displays hysteresis and thixotropy (resistance decreases with repeated movement) (Axelson and Hagbarth, 2001; Drake and Charles; Gillard et al., 2000; Pando et al.). 6) Wrist stiffness may be influenced by eccentric exercise completed prior to measurements (Leger and Milner, 2000; Whitehead et al., 2003; Whitehead et al., 2001).

The importance of these findings cannot be understated; however, we are still a long way away from a universal theory for wrist stiffness. The methodologies used in previous studies are inconsistent, differing on the definition of the neutral position, perturbation method, finger constraints, test velocity, and other factors. Much of this is expected as new studies uncover factors influencing wrist stiffness previously unknown to researchers. As the field progresses, 
however, the lessons learned from previous studies are not always fully appreciated nor are they always applied in new work. In addition to methodological gaps, the scope of previous studies has not been sufficient to characterize behavior over the complete ROM of the wrist, not to mention that there is an alarmingly large variability in the reports of 1D and 2D stiffness found in literature, with wrist stiffness values spanning several orders of magnitude.

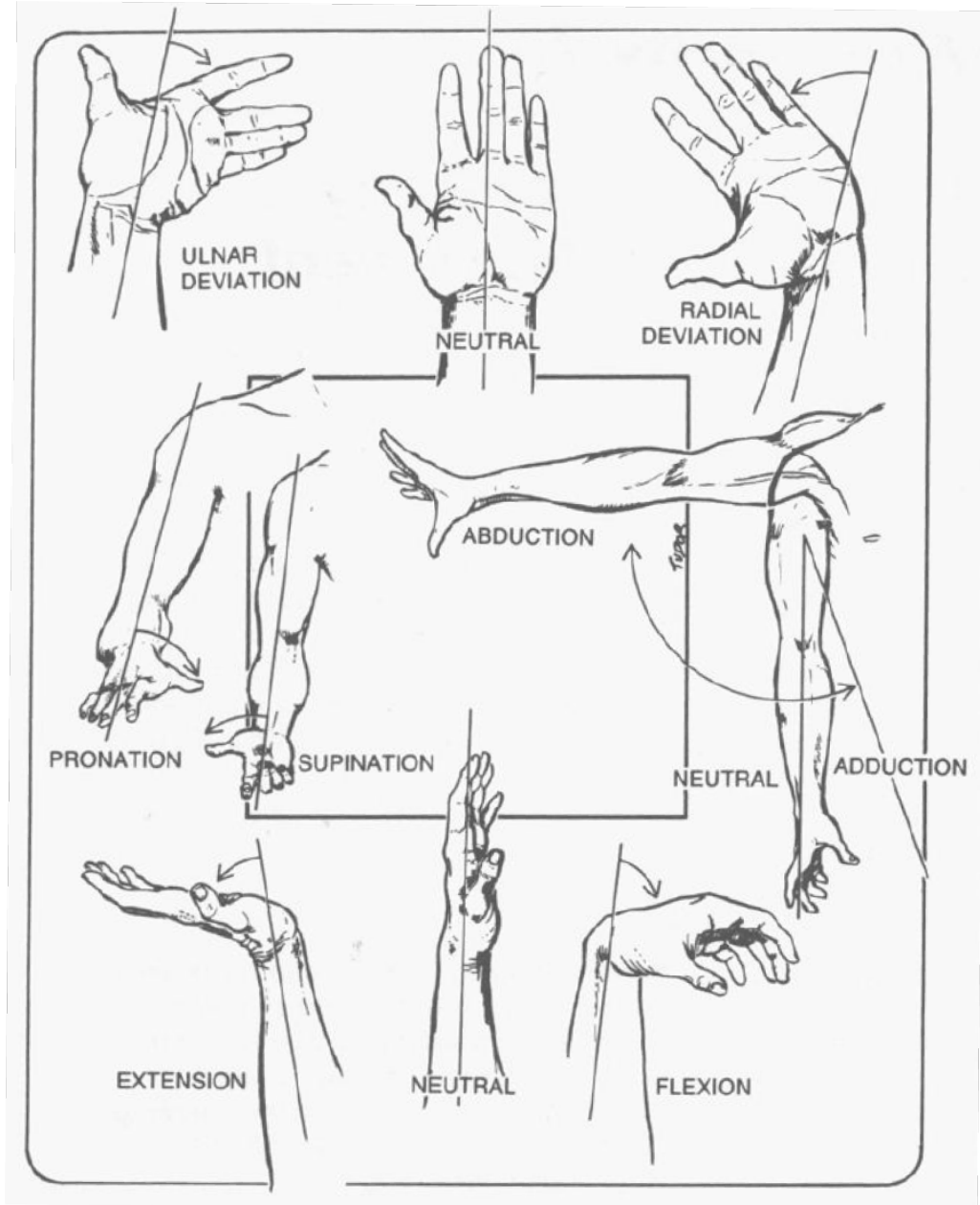

Figure 1-1: Definitions of wrist and forearm movement. (Jim Albers, 2003)

Activities of daily living commonly involve all three degrees-of-freedom (DOF) of the wrist and forearm); therefore, understanding the wrist joint requires a knowledge of the stiffness 
throughout the three-dimensional joint space of the wrist and forearm. Most previous studies of wrist or forearm stiffness, however, have measured passive joint stiffness in only 1 DOF, most often FE (Axelson and Hagbarth, 2001; De Serres and Milner, 1991; Gielen and Houk, 1984; Leger and Milner, 2000; Lehman and Calhoun, 1990). Few studies have measured stiffness in RUD (Rijnveld and Krebs, 2007), and only one study has measured stiffness in combinations of FE and RUD (Formica et al., 2012). Likewise, only a single study has measured the passive stiffness in PS (Formica et al., 2012). Here we present the passive stiffness (i.e. in the absence of muscle activity) of the wrist and forearm for $15^{\circ}$ movements involving combinations of PS, FE, and RUD.

The purpose of the present research is to characterize the passive stiffness of the wrist joint in all three DOF of wrist motion - wrist FE and RUD, as well as forearm PS - within the $\mathrm{ROM} \pm 15^{\circ}$ from the neutral orientation of the wrist. This knowledge will enable the characterization of how the wrist and forearm interact to produce healthy, graceful movement.

We begin with a thorough review of the current state of the literature with emphasis on all known factors influencing passive wrist joint stiffness and their quantified effects. Following the review, we present a detailed discussion of the methods developed and utilized in the present characterization. This is followed by the presentation and discussion of our central experiment. The final chapter provides a discussion of the research and results from a global perspective, identifying key areas of future research. 


\section{LITERATURE REVIEW}

A sound understanding of wrist dynamics - the forces and torques which the wrist encounters during motion - is an essential element in understanding and advancing the neurological, medical, and engineering fields associated with the wrist. For example, repetitive stress injury may result when the wrist joint is forced to make movements requiring high levels of torque. More accurate characterizations of the joint torques experienced by the wrist during activities of daily living will allow ergonomic designers to create workspaces and tools that allow users to avoid being forced into awkward, high-torque wrist motions.

Wrist dynamics are a composed of three primary torque sources: 1) inertia, 2) stiffness, and 3) damping effects. Because the present research deals solely with characterizing passive stiffness of the wrist joint, some justification is needed to explain the exclusion of inertia and damping.

Inertia plays a significant and dominating role in the dynamics of proximal joints; however, for the wrist joint, a distal joint, passive joint stiffness has been shown to have an even greater effect on joint dynamics than inertia - approximately ten times greater-for all but the fastest wrist movements (Charles and Hogan, 2011). The influence of passive wrist stiffness may be observed in the stereotyped curvature of active wrist movements (Charles and Hogan, 2012). In addition, passive wrist joint stiffness has been determined to be anisotropic in nature, meaning that the amount of passive stiffness encountered by the wrist varies with the direction of movement. For example, a movement in pure radial-ulnar deviation (RUD) must overcome 
nearly five times the stiffness that a similar movement would encounter in pure flexion-extension (FE) (Drake and Charles).

Damping is a velocity dependent phenomenon: the quicker the wrist movement, the higher the damping torque resisting movement. Little has been done to determine the magnitude of wrist damping relative to wrist stiffness during passive wrist rotations; however, damping has been shown to be much smaller than wrist stiffness during movements with active muscle contraction (Halaki et al., 2006; Milner and Cloutier, 1993, 1998). Having established the relative dominance of passive stiffness over inertia and damping effects, accurately modeling wrist joint dynamics requires characterization of passive joint stiffness throughout the joint's range-of-motion (ROM).

Over the years, a number of studies have been conducted to quantify passive stiffness in the wrist, approaching the problem from various angles, using differing methodologies and experimental methods. Their work has elucidated a number of key factors influencing the characterization of passive wrist stiffness - including tested range-of-motion, finger constraints, and test velocity, to name a few-all of which must controlled or otherwise accounted for in future studies.

Our purpose is to provide a holistic view of the current state of the literature, with careful treatment of all factors influencing passive wrist stiffness inasmuch as they have been reported and studied, and to indicate areas where future research is necessary to provide a unified model of passive wrist joint stiffness. We begin with a discussion of wrist ROM, in view of the fact that joint stiffness determines ROM, and continue with a presentation of the various factors which have been demonstrated to affect passive joint stiffness in the wrist. 
We begin with a brief explanation of the range of motion (ROM) and physiological contributions of various tissues to passive joint stiffness. Following the introductory material, we present the results of our inquiry by subtopic. Joint stiffness has been demonstrated to be a function of joint angle, physiological constraints, direction of movement (anisotropy), the orientation of kinematically linked body segments, test velocity (or strain-rate), exercise, muscle activation, repetition of movement (thixotropy), and the direction of movement (i.e. inbound or outbound) along a given path (hysteresis).

\subsection{Range of Motion (ROM)}

Wrist stiffness and ROM are directly related one to another: the range-of-motion that is available to the wrist is that region where the forearm muscles are sufficiently strong to overcome the wrist joint's naturally occurring stiffness. The wrist joint has two principle degrees-of-freedom: flexion-extension (FE) and radia-ulnar deviation (RUD). Pronation and supination of the wrist joint is minimal and not directly controlled by the neuromuscular system, hence it has not been included in our discussion. The wrist joint also experiences some translation (Patterson et al., 1998; Valero-Cuevas and Small, 1997), but this has been shown to be small (Kaufmann et al., 2005; Kaufmann et al., 2006).

While it is tempting to represent the wrist as a simple universal joint, the FE and RUD axes do not intersect; a number of studies have found that the FE axis of rotation is proximal to the RUD axis of rotation (Andrews and Youm, 1979; Brumbaugh et al., 1982; Goto et al., 2005; Horii et al., 1993; Kaufmann et al., 2005; Kaufmann et al., 2006; Leonard et al., 2005; Neu et al., 2001; Sommer and Miller, 1980; Youm et al., 1978). Additional studies have sought to distinguish between the flexion and extension axes and the radial deviation and ulnar deviation

axes. Sarrafian (Sarrafian et al., 1977) observed that the midcarpal joint accounted for $60 \%$ of 
flexion while the radio-carpal joint accounted for $66.5 \%$ of extension. Kobayashi et al. (Kobayashi et al., 1997) found that the proximal and midcarpal rows displace nearly the same amount in extension, while the distal carpal row flexes more than the proximal carpal row by a ratio of about 3-to-2. Ulnar deviation was observed to occur equally between the radiolunate and lunocapitate joints, while radial deviation occurred mainly in the lunocapitate joint (Kobayashi et al., 1997). MRI scans and computed tomography have reported that FE occurs primarily at the radiocarpal joint—between the radius and proximal row of carpal bones—while RUD occurs primarily at the midcarpal joint—-between the distal carpal bones and the proximal carpal bones (Goto et al., 2005; Kaufmann et al., 2005; Kaufmann et al., 2006).

The maximum or extreme ROM (x-ROM) of the wrist has long been an important clinical indication of the healthiness of the wrist joint and of the progress of individual's recovering from injury, stroke, or other wrist affliction. Tabulated results from selected x-ROM studies are presented in Table 2-1. The $\mathrm{x}-\mathrm{ROM}$ are presented as combined flexion-extension (FE) and radioulnar deviation (RUD) ranges and further subdivided into flexion (F), extension (E), radial deviation (RD), and ulnar deviation (UD) contributions.

When comparing $\mathrm{x}$-ROM studies it is important to take note of the specific measurement techniques used by the researchers. Differences between the definition of the neutral position, the presence of constraints on finger motion, or the method of displacing the wrist (e.g. passive circumduction performed by the researcher, active circumduction performed by the patient, or ROM achieved by a specified torque threshold) can significantly influence the results. It is not uncommon to define neutral FE to be when the long axis of the forearm and the third metacarpal (MET3) are aligned; however, it is also common to define neutral FE as the orientation of the wrist when the hand is gripping a handle in line with the long axis of the forearm. In this case, 
the neutral position of the handle-based study is in $20^{\circ}$ extension relative to the MET3-based study. Notwithstanding the protocol differences, a rough consensus may be reached that the wrist joint's x-ROM has approximately $134^{\circ}$ of $\mathrm{FE}\left(66^{\circ} / 68^{\circ}\right)$ and approximately $60^{\circ}$ of $\mathrm{RUD}$ $\left(39^{\circ} / 21^{\circ}\right)$

Table 2-1: Selected results from extreme range-of-motion (x-ROM) wrist studies, including results from passive ROM and active ROM studies. The mean was obtained as a straight average without compensating for the number of participants in each study.

All units in degrees.

\begin{tabular}{|c|c|c|c|c|c|c|c|c|c|c|}
\hline \multirow[b]{2}{*}{$\begin{array}{l}\text { (Boone and Azen, 1979) } \dagger \text { All } \\
\text { ages }\end{array}$} & \multirow{2}{*}{$\begin{array}{c}\mathbf{F E} \\
149\end{array}$} & \multicolumn{2}{|c|}{ F (\%) } & \multicolumn{2}{|c|}{ E (\%) } & \multirow{2}{*}{$\begin{array}{c}\text { RUD } \\
56\end{array}$} & \multicolumn{2}{|c|}{ UD (\%) } & \multicolumn{2}{|c|}{ RD (\%) } \\
\hline & & 75 & $(50 \%)$ & 74 & $(50 \%)$ & & 35 & $(63 \%)$ & 21 & $(38 \%)$ \\
\hline $\begin{array}{l}\text { (Boone and Azen, 1979) } \dagger \leq 19 \\
\text { yrs. }\end{array}$ & 154 & 78 & $(51 \%)$ & 76 & $(49 \%)$ & 59 & 37 & $(63 \%)$ & 22 & $(37 \%)$ \\
\hline $\begin{array}{l}(\text { Boone and Azen, 1979) } \dagger>19 \\
\text { yrs. }\end{array}$ & 149 & 75 & $(50 \%)$ & 74 & $(50 \%)$ & 57 & 36 & $(63 \%)$ & 21 & $(37 \%)$ \\
\hline (Crisco et al., 2011) & 129 & 65 & $(50 \%)$ & 64 & $(50 \%)$ & 63 & 42 & $(67 \%)$ & 21 & $(33 \%)$ \\
\hline (AAOS, 1965) & 144 & 73 & $(51 \%)$ & 71 & $(49 \%)$ & 52 & 33 & $(63 \%)$ & 19 & $(37 \%)$ \\
\hline$(\mathrm{Li}, 2002) \dagger \S$ & 144 & 75 & $(52 \%)$ & 69 & $(48 \%)$ & 75 & 51 & $(68 \%)$ & 24 & $(32 \%)$ \\
\hline (Li et al., 2005) $† \S$ Isolated $D O F$ & 108 & 41 & $(38 \%)$ & 67 & $(62 \%)$ & 55 & 35 & $(64 \%)$ & 20 & $(36 \%)$ \\
\hline $\begin{array}{l}\text { (Li et al., 2005) } † \S \\
\text { Circumduction }\end{array}$ & 100 & 36 & $(36 \%)$ & 64 & $(64 \%)$ & 52 & 32 & $(62 \%)$ & 20 & $(38 \%)$ \\
\hline (Marshall et al., 1999) $† \S$ & 140 & 67 & $(48 \%)$ & 73 & $(52 \%)$ & 69 & 47 & $(69 \%)$ & 21 & $(31 \%)$ \\
\hline (Marshall et al., 1999) $\$ \S$ & 152 & 72 & $(48 \%)$ & 79 & $(52 \%)$ & 66 & 46 & $(69 \%)$ & 21 & $(31 \%)$ \\
\hline (Ryu et al., 1991a) & 138 & 79 & $(57 \%)$ & 59 & $(43 \%)$ & 58 & 38 & $(66 \%)$ & 20 & $(34 \%)$ \\
\hline (Salvia et al., 2000) & 104 & 56 & $(54 \%)$ & 48 & $(46 \%)$ & 54 & 35 & $(65 \%)$ & 19 & $(35 \%)$ \\
\hline Mean of Studies & 134 & 66 & $(49 \%)$ & 68 & $(51 \%)$ & 60 & 39 & $(65 \%)$ & 21 & $(35 \%)$ \\
\hline
\end{tabular}

Inasmuch as joint stiffness is the determining factor in $\mathrm{x}-\mathrm{ROM}$, it is important to note the result reported by Boone and Azen (Boone and Azen, 1979) that X-ROM decreased with age (FE $\mathrm{x}-\mathrm{ROM}$ and RUD $\mathrm{x}-\mathrm{ROM}$ both decreased by 3\%). Though the differences are slight, they 
indicate an increase in passive joint stiffness with age. This particular factor in passive wrist stiffness requires additional examination.

Additional studies on wrist $\mathrm{x}-\mathrm{ROM}$ that published partial measurements-i.e. either single DOF measurements or combined FE and combined RUD x-ROMs without sub-DOF divisions - may be found in (Brumfield et al., 1966; Gunal et al., 1996; Ojima et al., 1992; Palmer et al., 1985; Wagner, 1988). Wagner (Wagner, 1988) includes a statistical analysis of xROM in RUD for pianists, comparing the results between males and females as well as between right and left hands.

While an accurate description of the wrist's $\mathrm{x}-\mathrm{ROM}$ is important, the majority of activities of daily living (ADL) occur within a much smaller functional ROM (f-ROM). The fROM is typically determined by measuring the wrist angles used while completing specified activities. As with the $\mathrm{x}-\mathrm{ROM}$, the $\mathrm{f}-\mathrm{ROM}$ is highly dependent upon passive stiffness in the wrist. It is suspected that one of the factors influencing the f-ROM used by the neuromuscular system is the effort required to overcome the passive stiffness of the wrist. The f-ROM, therefore, gives a sense of the effort that the neuromuscular system deems appropriate. These activities can apply either to a general population or to a desired target population. As with the values reported by $\mathrm{x}-\mathrm{ROM}$ studies, the values reported by $\mathrm{f}-\mathrm{ROM}$ studies are subject to their respective experimental protocols. In addition, the type of ADLs tested by the study will affect the $\mathrm{f}-\mathrm{ROM}$ necessary to accomplish them; for example, rising from a chair requires extreme wrist extension, whereas typing on a keyboard requires relatively little. Values from f-ROM studies are typically reported either as the mean ROM used to accomplish the given ADLs or as the minimal ROM necessary (the term minimal may be misleading in that the minimal f-ROM 
generally represents the combination of the highest average values for F, E, RD, and UD observed when averaging the results of each ADL separately).

A third characterization of wrist range-of-motion, even smaller than the f-ROM, has been described in literature. Using restrictive wrist splints, Nelson (Nelson, 1997) demonstrated that 123 ADLs could be successfully accomplished with only $5^{\circ}$ flexion, $6^{\circ}$ extension, $7^{\circ}$ radial deviation, and $6^{\circ}$ ulnar deviation. Of the 123 ADLs that could be successfully completed, 116 were accomplished with a minimal degree of difficulty. This characterization may be deemed the minimal ROM (m-ROM), but it should not be confused with the minimal f-ROM.

It has been argued (Nelson, 1997) that the m-ROM should drive ROM-based discussions and research because it describes the range actually needed to perform ADLs and not just the total possible range of the joint (x-ROM) or the range used to accomplish ADLs (f-ROM) them. Owing to the redundant nature of the human arm, the loss or restriction of one or more DOF may be compensated for by increased activity in the remaining joints. An in-depth examination of the long-term implications of being limited to the m-ROM must be conducted to determine 1) whether the successfully performed ADLs actually required no more ROM than what the wrist braces allowed or if the restriction was compensated for by the remaining DOF of the arm, and 2) whether the compensating joints incur serious detrimental effects as a result.

Short-term studies where the participants' wrists were immobilized by wrist braces found a significant increase in the time to complete given tasks (Adams et al., 2003; Carlson and Trombly, 1983; Chan and Chapparo, 1999; King et al., 2003; Pagnotta et al., 2005), significant increases in shoulder and torso movement (Chan and Chapparo, 1999; King et al., 2003; MayLisowski and King, 2008; Mell et al., 2005; Shu and Mirka, 2006), significantly higher muscle activity in the forearm, upper arm, and shoulder muscles (Bulthaup et al., 1999; Mell et al., 2006; 
Shu and Mirka, 2006; Yoo et al., 2010), and that movements were less direct and less smooth (King et al., 2003). Patients also reported mild fatigue of the shoulder and upper trunk following tasks involving wrist immobilization (Carlson and Trombly, 1983).

Table 2-2: Results from functional range-of-motion (f-ROM) wrist studies. Results marked "mean f-ROM" represent the average $\mathrm{ROM}$ used throughout the entire study. Results marked "minimal f-ROM" represent the highest single-DOF ranges calculated when averaging each ADL separately. The "mean of studies" represents a straight average without weighting.

\begin{tabular}{|c|c|c|c|c|c|c|c|c|c|c|}
\hline & FE & \multicolumn{2}{|c|}{ F (\%) } & \multicolumn{2}{|c|}{ E (\%) } & \multirow{2}{*}{$\begin{array}{c}\text { RUD } \\
41\end{array}$} & \multicolumn{2}{|c|}{ UD (\%) } & \multicolumn{2}{|c|}{ RD (\%) } \\
\hline$($ Aizawa et al., 2010) $\dagger$ & 105 & 76 & $(72 \%)$ & 29 & $(28 \%)$ & & 33 & $(80 \%)$ & & $(20 \%)$ \\
\hline $\begin{array}{l}\text { (Brumfield and Champoux, } \\
\text { 1984) } \dagger\end{array}$ & 82 & 19 & $(23 \%)$ & 63 & $(77 \%)$ & -- & -- & -- & -- & -- \\
\hline $\begin{array}{l}\text { (Brumfield and Champoux, } \\
\text { 1984) }\end{array}$ & 45 & 10 & $(22 \%)$ & 35 & $(78 \%)$ & -- & -- & -- & -- & -- \\
\hline (Palmer et al., 1985) & 35 & 5 & $(14 \%)$ & 30 & $(86 \%)$ & 25 & 15 & $(60 \%)$ & 10 & $(40 \%)$ \\
\hline (Ryu et al., 1991b) $\dagger$ & 114 & 54 & $(47 \%)$ & 60 & $(53 \%)$ & 57 & 40 & $(70 \%)$ & 17 & $(30 \%)$ \\
\hline (van Andel et al., 2008) $\dagger$ & 130 & 66 & $(51 \%)$ & 64 & $(49 \%)$ & -- & -- & -- & -- & -- \\
\hline $\begin{array}{l}\text { Swanson (Carlson and Trombly, } \\
\text { 1983) }\end{array}$ & 20 & 10 & $(50 \%)$ & 10 & $(50 \%)$ & -- & & -- & -- & -- \\
\hline $\begin{array}{l}\text { Keller (Carlson and Trombly, } \\
\text { 1983) }\end{array}$ & 100 & 60 & $(60 \%)$ & 40 & $(40 \%)$ & -- & -- & -- & -- & -- \\
\hline Mean of Studies & 81 & 37 & $(38 \%)$ & 47 & $(62 \%)$ & 41 & 29 & $(70 \%)$ & 12 & $(30 \%)$ \\
\hline
\end{tabular}

Reporting $\mathrm{x}-\mathrm{ROM}$ and $\mathrm{f}-\mathrm{ROM}$ with values for $\mathrm{F}, \mathrm{E}, \mathrm{RD}$, and UD may lead one to surmise that the wrist's ROM resembles a perfect rectangle, each of the four corners being determined by a Cartesian pair such as (F, RD). In reality, however, a trace of the wrist's ROM during circumduction has a very different shape. As seen in Figure 2-1, the wrist's ROM is coupled between FE and RUD: the extreme value of FE depends on the wrist's RUD position, and vice versa. 


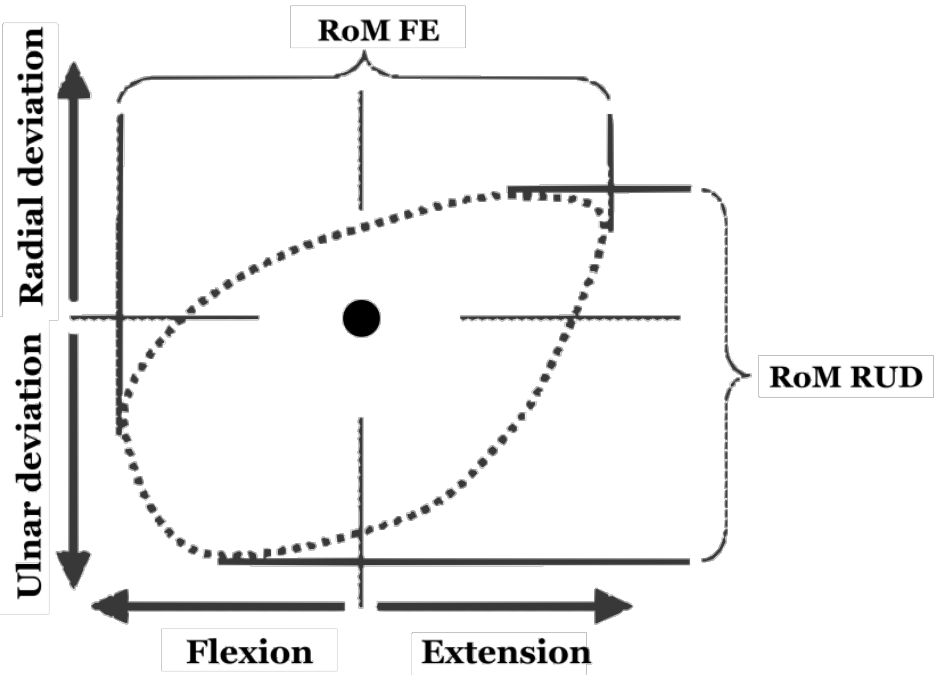

Figure 2-1: Stereotypical plot of wrist ROM throughout circumduction. Note the interdependence of FE and RUD ROMs-e.g. extreme RD is much higher in extension than it is in flexion. (Gehrmann et al., 2008).

\subsection{Definition of Neutral Position and Arm Orientation}

Defining the neutral position (or zero position) is a critical step for any characterization of joint stiffness. With regard to the wrist joint, the researcher must specify the neutral position for FE and RUD of the wrist, pronation-supination (PS) of the forearm, and abduction-adduction of the shoulder. While the necessity of prescribing the origin of FE and RUD may be obvious, it may not be quite so apparent why prescribing the initial orientations of the remaining joints are so critical.

Forearm orientation may significantly affect the passive stiffness of the wrist joint due to the fact that the muscles controlling wrist motion cross multiple joints. De Rugy et al. (de Rugy et al., 2012) reported that forearm rotation counter-rotated the pulling direction of the wrist muscles by $12 \%$ relative to the wrist. Musculature has been reported to contribute roughly $40 \%$ of passive stiffness for feline wrist (Johns and Wright, 1962); it is possible that forearm alignment in PS could significantly impact measured wrist stiffness. This observation takes on 
critical importance when one notes that the definition of the neutral position for the forearm can vary greatly between wrist stiffness studies.

The International Society of Biomechanics (Wu et al., 2005) defines the neutral position of the forearm to be when the elbow is flexed $90^{\circ}$ and the thumb is pointed at the shoulder. In practice, the authors have found that aligning the dorsal aspects of the radial tubercle and the ulnar head with a vertical plate provides a more repeatable definition for neutral pronationsupination. Another frequently used practice is to define the forearm as being in neutral rotation when the hand is grasping a vertical handle. It has recently been reported that orienting the hand and wrist via vertical handle results in $6^{\circ}$ of pronation at the neutral position when compared to an orientation where the dorsal aspects of the radial tubercle and ulnar head are aligned vertically (Drake and Charles). The difference may be further exaggerated if compared to other definitions of the neutral position.

Whether using the ISB definition of neutral forearm position, the vertical plate method, a vertical handle, or other technique, the orientation of the shoulder in abduction-adduction may also significantly affect passive wrist stiffness. So long as the wrist is constrained to be in a roughly vertical orientation, shoulder abduction results in nearly equivalent supination of the forearm. This is a highly relevant fact when one notes that reported values for shoulder abduction range between $0^{\circ}$ and $40^{\circ}$ across studies (Drake and Charles; Formica et al., 2012). Even studies that define neutral forearm rotation in the same manner may be characterizing wrist stiffness under significantly different conditions if the neutral position of the shoulder is not consistent as well.

A definitive characterization of the effects of the definition of neutral position of the wrist, forearm, and shoulder has not yet been reported. 


\subsection{Movement Constraints}

Gehrmann et al. (Gehrmann et al., 2008) conducted a novel study in which they measured the active $\mathrm{x}-\mathrm{ROM}$ with four different finger constraint conditions: 1) unconstrained, 2) holding a 50 mm diameter cylinder, 3) holding a $25 \mathrm{~mm}$ diameter cylinder, and 4) clenched fist. They found that the ROM for the unconstrained finger condition was significantly greater than the ROM values for the constrained conditions in FE and RUD $(p<0.01)$, and the ROM values for the cylinder constraints were significantly greater than the ROM values for clenched fist $(p<0.01)$. The difference between ROM values for the large cylinder and the small cylinder, however, was not significant. Taken separately, flexion x-ROM was significantly affected by finger constraint $(\mathrm{p}<0.01)$, whereas extension $\mathrm{x}-\mathrm{ROM}$ showed little correlation to finger constraint. Similarly, radial deviation $\mathrm{x}-\mathrm{ROM}$ was not significantly affect by finger constraint $(\mathrm{p}>0.15)$, whereas ulnar deviation $\mathrm{x}-\mathrm{ROM}$ was significantly affected $(\mathrm{p}<0.05)$.

As compared to the unconstrained condition, constraining the subject's fingers to the large cylinder, small cylinder, or clenched fist condition had the effect of decreasing the wrist's ROM by $13 \%, 16 \%$, and $27 \%$ in flexion respectively, and by $10 \%, 10 \%$, and $11 \%$ in ulnar

deviation respectively [See Table 2-3]. Gehrmann et al. (Gehrmann et al., 2008) attribute the decrease in flexion ROM to increased passive resistance from the elongated finger extensors and decreased force production capability due to elongation of the wrist flexors. The decrease in ulnar deviation ROM was attributed to increased passive resistance from the elongated finger abductor and extensor muscles.

The findings reported in Gehrmann et al. (Gehrmann et al., 2008) are of critical importance to those attempting to characterize the passive stiffness of the wrist joint because it demonstrates the highly significant effect that methodology—specifically finger constraints- 
have on results. We know of no study in passive wrist stiffness that directly parallels the methodology of Gehrmann et al. (Gehrmann et al., 2008); however, because of the direct connection between wrist stiffness and x-ROM, it may be reasonably safe to assume that finger constraints will significantly affect the values measured during passive wrist studies at or below the level reported for $\mathrm{x}-\mathrm{ROM}$.

Table 2-3: Wrist $x-R O M$ as measured through active circumduction with the fingers

1) unconstrained, 2) grasping a 50mm diameter cylinder, 3) grasping a $25 \mathrm{~mm}$ diameter cylinder, and 4) clenched in a fist. Values taken from (Gehrmann et al., 2008)

\begin{tabular}{|l|c|c|c|c|}
\cline { 2 - 5 } \multicolumn{1}{c|}{} & $\mathbf{1}$ & $\mathbf{2}$ & $\mathbf{3}$ & $\mathbf{4}$ \\
\hline Flexion & $151^{\circ} \pm 13^{\circ}$ & $140^{\circ} \pm 10^{\circ}$ & $142^{\circ} \pm 14^{\circ}$ & $130^{\circ} \pm 10^{\circ}$ \\
\hline Extension & & & & \\
\cline { 1 - 5 } Radial Deviation & $80^{\circ} \pm 9^{\circ}$ & $74^{\circ} \pm 12^{\circ}$ & $73^{\circ} \pm 10^{\circ}$ & $73^{\circ} \pm 14^{\circ}$ \\
\hline
\end{tabular}

\subsection{Physiological Contributions}

Accounting for the factors influencing passive joint stiffness requires a sound understanding of the relative contributions of various tissues to both passive wrist torque and passive wrist stiffness. Noting the rheological similarities between the human metacarpophalangeal joint and the feline wrist, Johns and Wright (Johns and Wright, 1962) conducted a study to determine the relative contributions of skin, muscles, tendons, and the joint capsule to wrist stiffness. Throughout the measured range the joint capsule provided the most significant contribution to overall torque at $47 \%$; the joint capsule contributed $41 \%$, the tendons $10 \%$, and the skin $2 \%$. Though their results do not apply directly to the wrist joint, they give a sense of the importance of each of the tissues surrounding a joint. It is hoped that a similar study may one day be performed on the human wrist. 


\subsection{Joint Angle}

Passive wrist stiffness has been shown to vary non-linearly with increasing joint angle [Figure 22]. Upon initial displacement from a period of rest, the wrist passes first through a region of relatively high stiffness (from the origin to A). Wrist stiffness then decreases significantly as the joint enters a region of roughly linear stiffness through the midrange of movement (from A to B). As the joint is displaced towards the extreme bounds of the ROM, the stiffness increases again at a dramatic rate until movement of the joint is arrested (from B to C). Reversing the direction of movement, the stiffness decreases quickly and the torque between $\mathrm{D}$ and $\mathrm{E}$ falls below the level from A to B (See section on Hysteresis). The stiffness then holds constant at approximately the same value as that of the left-to-right movement (D to E). As the joint approaches the extreme ROM on the left, the stiffness again increases dramatically until movement is arrested (E to F). If the movement is continued from $\mathrm{F}$ to $\mathrm{C}$, the torque response will follow the top curve, paralleling the path just taken.

As noted above, the stiffness observed during the initial phase of a movement, when muscle is elongated less than $1 \%$ of its total length, is generally higher than that seen during the remainder of the movement. Upon observing this phenomenon, D. K. Hill (Hill, 1968) coined the term "short range elastic component (SREC)". A few years later, Rack and Westbury (Rack and Westbury, 1974) referred to it as "short range stiffness (SRS)", and the two terms have been used more or less interchangeably ever since. SREC or SRS has been found to exist in striated muscle and cardiac muscle whether activated or relaxed (Campbell, 2010). 


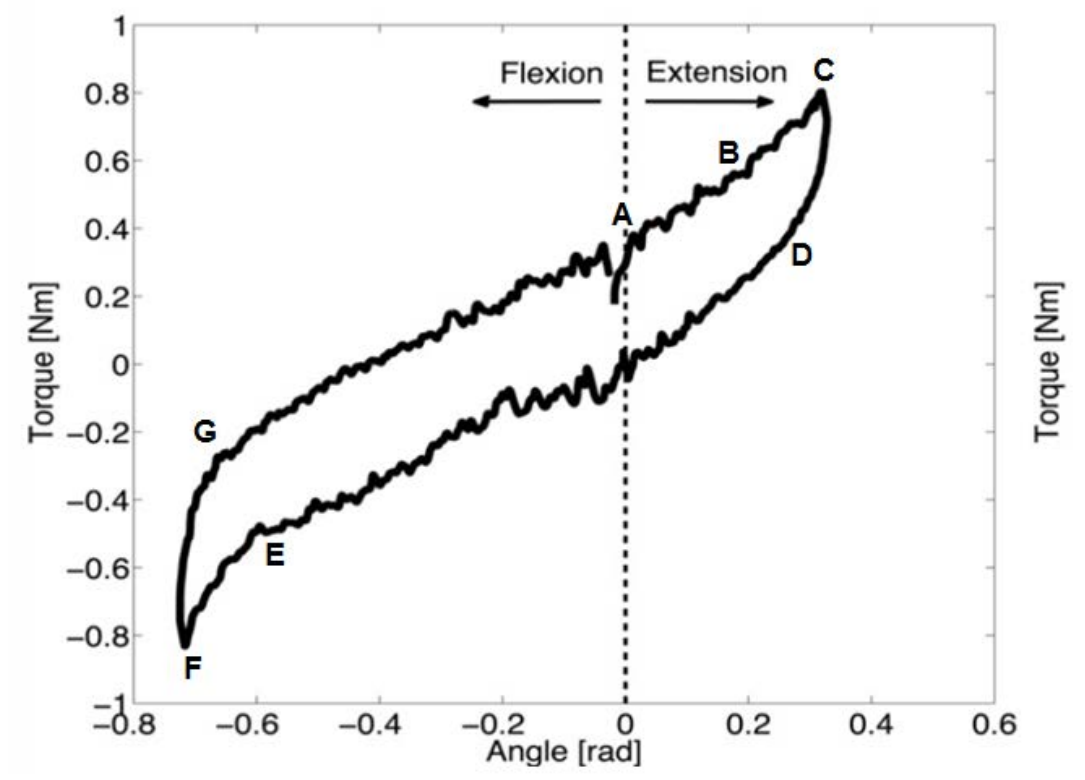

Figure 2-2: Torque response for passive repetitive adduction and abduction of the metacarpophalangeal joint. The characteristics of this plot are similar to those found in torque response plots of the wrist joint. Adapted from (Formica et al., 2012).

In a study of human motor control of the wrist joint, Axelson and Hagbarth reported seeing the SRS effect over the first 3-4 $4^{\circ}$ of passive wrist movements [Figure 2-3] (Axelson and Hagbarth, 2001). Over the initial $2^{\circ}$ of motion, the wrist experiences SRS on the order of 1.3 $\mathrm{Nm} / \mathrm{rad}$. From $2^{\circ}-15^{\circ}$, the wrist experiences roughly linear midrange stiffness on the order of 0.2 $\mathrm{Nm} / \mathrm{rad}$, less than one-fifth the magnitude during the SRS region. Van Eesbeek et al. (van Eesbeek et al., 2010) reported SRS of $7.3 \pm 2.8 \mathrm{Nm} / \mathrm{rad}$ in flexion and $7.5 \pm 4.9 \mathrm{Nm} / \mathrm{rad}$ in extension throughout the range of $\pm 2^{\circ}$. As the strain rate increases, the SRS phenomenon peaks earlier during the movement; however, the slope of the torque-position curve does not change (Campbell, 2010). For thorough treatment of SRS phenomenon in general, the reader should refer to Campbell (Campbell, 2010). 


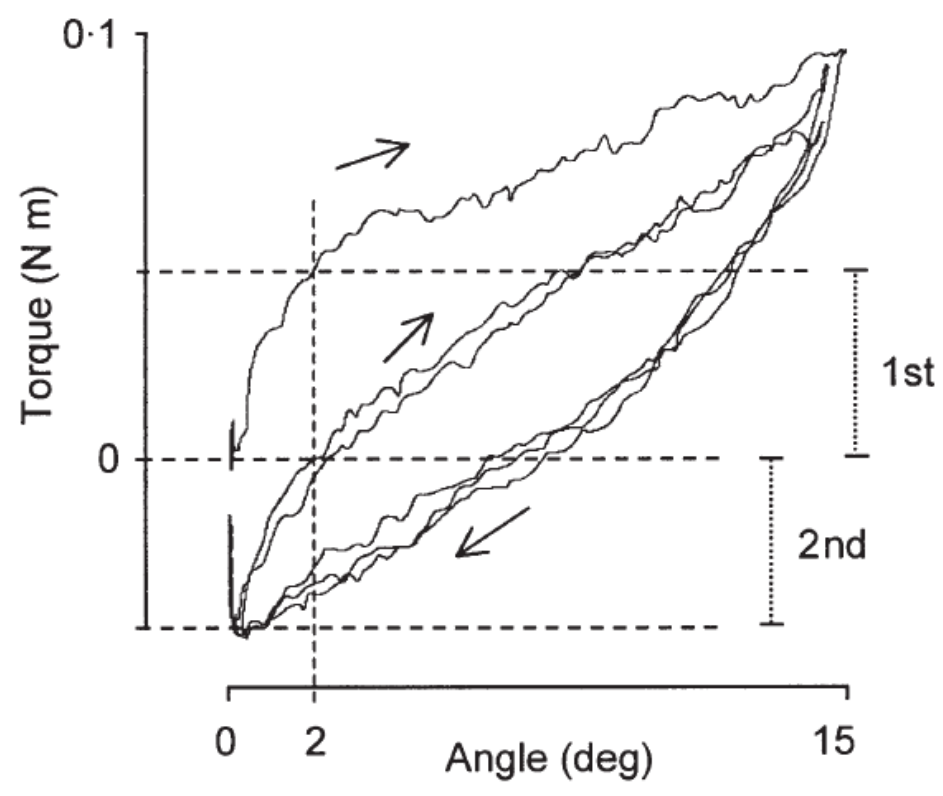

Figure 2-3: Torque response for passive repetitive extension and flexion of the wrist joint. The SRS phenomenon is clearly seen in the first $2^{\circ}$ of movement. (Axelson and Hagbarth, 2001).

\subsection{Anisotropy}

Passive wrist stiffness is anisotropic in nature, which means that the amount of stiffness experienced by the wrist joint depends on the direction in which the wrist is moving-i.e. flexion, extension, radial deviation, ulnar deviation, or combinations. Furthermore, wrist stiffness has been shown to be coupled, such that a movement in FE produces a reaction torque in RUD as well as in FE, and vice versa (Charles and Hogan, 2011; Drake and Charles; Formica et al., 2012). Evidence of the coupled nature of wrist stiffness may be seen in the wrist circumduction plot in Figure 2-1; the $\mathrm{x}-\mathrm{ROM}$ of the wrist depends both on the amount of FE and the amount of RUD being used.

The vast majority of studies that have been performed to determine passive wrist stiffness examined only one of the directions of wrist movement [See Table 2-3]. If a linear approximation of stiffness is taken (a generalization that has been shown to be appropriate for movements on the order of $\pm 15^{\circ}$ (Charles and Hogan, 2011; Drake and Charles; Formica et al., 
2012)) the nonlinear stiffness field may be reduced to a $2 \times 2$ linear stiffness tensor. The main diagonal terms of the tensor represent the stiffness encountered in flexion/extension (FE) with movement in $\mathrm{FE}\left[\mathrm{K}_{\mathrm{FE}}\right]$ and the stiffness encountered in radial/ulnar deviation (RUD) with movement in RUD [ $\left.\mathrm{K}_{\mathrm{RUD}}\right]$. The off-axis terms represent the coupled stiffness in FE with movement in RUD [ $\left.\mathrm{K}_{\mathrm{FE} / \mathrm{RUD}}\right]$ and the coupled stiffness in RUD with movement in FE [K $\left.\mathrm{K}_{\mathrm{RUD} / \mathrm{FE}}\right]$. It has been shown that the anti-symmetric portion of the linear stiffness tensor is negligible when compared to the symmetric portion (Charles and Hogan, 2011; Drake and Charles; Formica et al., 2012); hence, the anti-symmetric portion may be discarded to simplify the result and to facilitate graphical representation. For the remaining symmetric tensor, the off-axis terms-i.e. the $\mathrm{K}_{\mathrm{FE} / \mathrm{RUD}}$ and $\mathrm{K}_{\mathrm{RUD} / \mathrm{FE}}$ terms - are identical. (Hogan, 1985; Mussa-Ivaldi et al., 1985)

Historically, estimates for wrist stiffness in $\mathrm{K}_{\mathrm{FE}}$ have varied widely over several orders of magnitude from $0.002 \mathrm{Nm} / \mathrm{rad}$ (Lehman and Calhoun, 1990) to $22.92 \mathrm{Nm} / \mathrm{rad}$ (Crisco et al., 2011). While some of the discrepancies can be accounted for in methodological differences (e.g. Halaki et al. (Halaki et al., 2006) used strain rates of up to $407^{\circ}$ /second, whereas Formica et al. used strain rates of $5 \%$ second (Formica et al., 2012)), the range of reported values is still quite large. Recent studies indicate a much smaller range of values for $\mathrm{K}_{\mathrm{FE}}$ of 0.73 to $1.42 \mathrm{Nm} / \mathrm{rad}$ (Drake and Charles; Formica et al., 2012; Pando et al.).

Stiffness in RUD has been found to be significantly higher than stiffness in $\mathrm{K}_{\mathrm{FE}}$. The same studies reported values for $\mathrm{K}_{\mathrm{RUD}}$ of 1.49 and $2.78 \mathrm{Nm} / \mathrm{rad}$ and calculated that $\mathrm{K}_{\text {RUD }}$ was between 1.3-2.9 times larger than $\mathrm{K}_{\mathrm{FE}}$ (Drake and Charles; Formica et al., 2012; Pando et al.).

Coupled stiffness in the wrist has been shown to be small in comparison with noncoupled stiffness, but the effect is not so small as to be negligible. The $\mathrm{K}_{\mathrm{FE} / \mathrm{RUD}}$ terms, which represent the coupled stiffness, have been measured in the range -0.04 to $-0.27 \mathrm{Nm} / \mathrm{rad}$ (Drake 
and Charles; Formica et al., 2012; Pando et al.). By comparing the coupled stiffness terms ( $\mathrm{K}^{-}$ FE/RUD) with the non-coupled stiffness terms ( $\mathrm{K}_{\mathrm{FE}}$ and $\left.\mathrm{K}_{\mathrm{RUD}}\right)$, we see that the coupled stiffness $\mathrm{K}^{-}$ FE/RUD is between -0.05 and -0.31 times as large as $\mathrm{K}_{\mathrm{FE}}$ and between -0.02 and -0.13 times as large as $\mathrm{K}_{\mathrm{RUD}}$ [See Table 2-4].

While pronation-supination (PS) of the wrist (not to be confused with PS of the forearm) is not controlled by the neuromuscular system, we present one reference on the passive stiffness of the wrist joint in rotations. Ritt et al. (Ritt et al., 1996) examined the passive stiffness of the radiocarpal joint in cadavers over the ROM achieved by a maximum torque of $2.3 \mathrm{Nm}$. They measured passive stiffness of $1.72 \mathrm{Nm} / \mathrm{rad}$ within the midrange of wrist PS (achieved by a maximum torque of $\pm 0.2 \mathrm{Nm}$ ) and stiffness of $13.46 \mathrm{Nm} / \mathrm{rad}$ at the $\mathrm{x}-\mathrm{ROM}$ tested (achieved by a maximum torque of $\pm 2.3 \mathrm{Nm})$.

Table 2-4: Reported values of passive wrist stiffness. The values were taken over a range of wrist deflections, strain rates, finger constraints, and maximum applied forces, the exact magnitudes of which were seldom reported.

All values in Nm/rad.

\begin{tabular}{|l|c|c|c|}
\cline { 2 - 4 } \multicolumn{1}{c|}{} & FE/FE & RUD/RUD & RUD/FE \\
\hline (Rijnveld and Krebs, 2007) & -- & 1.45 & -- \\
\hline (Lehman and Calhoun, 1990) & $0.002-0.015$ & -- & -- \\
\hline (Axelson and Hagbarth, 2001) & 0.34 & -- & -- \\
\hline (Gielen and Houk, 1984) & $0.32-0.70$ & -- & -- \\
\hline (Drake and Charles) $\dagger$ & 0.73 & 2.16 & -0.04 \\
\hline (Pando et al.) & 0.85 & 2.02 & -0.27 \\
\hline (Formica et al., 2012) $\dagger$ & 0.96 & 1.49 & -0.16 \\
\hline (Drake and Charles) $\dagger$ & 0.96 & 2.78 & -0.09 \\
\hline (Formica et al., 2012) $\dagger$ & 1.42 & 1.85 & -0.19 \\
\hline (Leger and Milner, 2000) & 2.2 & -- & -- \\
\hline (De Serres and Milner, 1991) & 3 & -- & -- \\
\hline (van Eesbeek et al., 2010) & 3.05 & -- & -- \\
\hline
\end{tabular}

$\dagger$ Male subjects, $\ddagger$ Female subjects 


\subsection{Repetition of Movement (Thixotropy)}

The term 'thixotropy' is used to describe a change in material properties that occurs with repetition. Thixotropy is observed in wrist torque/position plots, where the torque required to complete a specified movement decreases with increasing repetitions. Axelson and Hagbarth (Axelson and Hagbarth, 2001) report an $18 \%$ decrease $(\mathrm{p}<0.01)$ in the torque required to complete the first and second repetitions of $15^{\circ}$ wrist extensions (See Figure 2-4). The thixotropic torque reduction was observed primarily between the first and second repetitions and did not continue throughout subsequent repetitions of the movement. After a 15 second rest period, the thixotropic effect was observed again. It should be kept in mind, that the thixotropic effect was reported for the torque measured only and not for the stiffness measured.

In their study, Drake and Charles (Drake and Charles) repeated $\pm 15^{\circ}$ movements three times in succession and compared the stiffness measured during each repetition. They found no statistically significant difference between the stiffness of that occurred during the first repetition and either of the subsequent repetitions. The reader is referred to Proske (Proske et al., 1993) for a thorough treatment of the general phenomenon . 


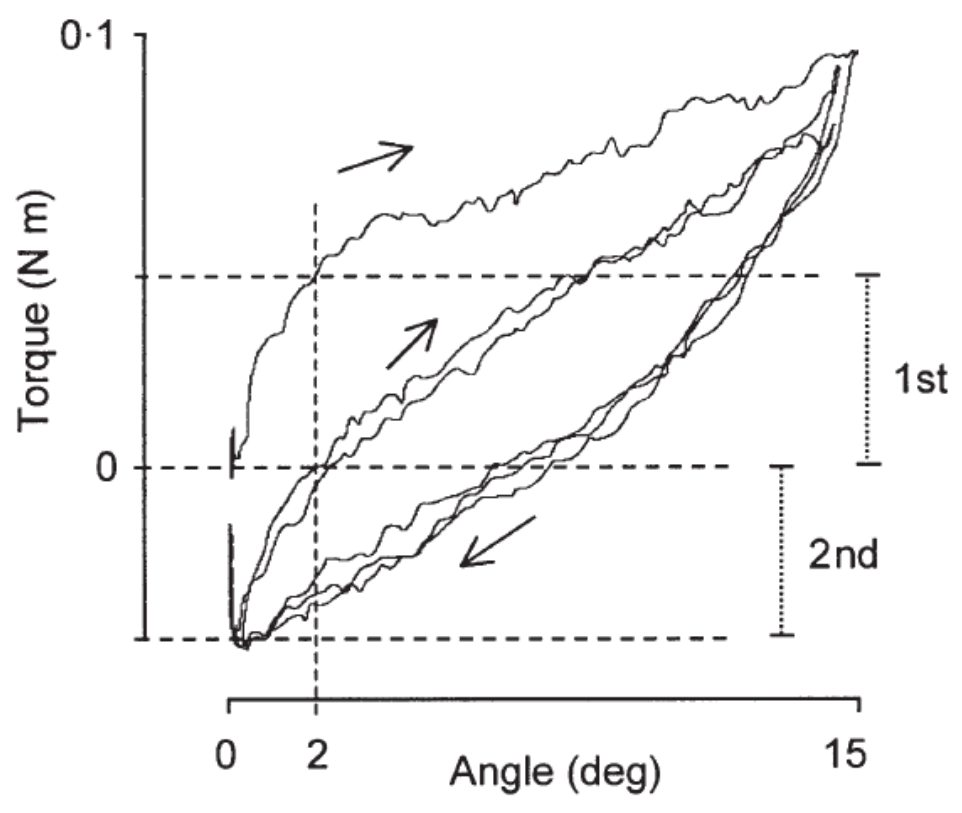

Figure 2-4: Torque responses to passive repetitive wrist extensions exceeding the limit for the shortrange stiffness. Note the thixotropic torque decrease between the first and second repetitions and hysterisis. Nm/rad. (Axelson and Hagbarth, 2001)

\subsection{Hysteresis}

Hysteresis is a property observed in torque-position plots in joints, muscles, tendons, and other biological tissues. Description of the phenomenon is best achieved graphically. Figure 2-4 is taken from Axelson and Hagbarth (Axelson and Hagbarth, 2001) and depicts the successive wrist extensions from the neutral position (on the left) to $15^{\circ}$ extension (on the right) and back again. The reader will observe that the torque required to displace the wrist from left to right is higher than that required to return the wrist from right to left to its original starting point. The phenomenon extends to the full ROM of the wrist joint from extreme flexion to extreme extension (and from extreme ulnar deviation to extreme radial deviation) as observed in the stereotyped depiction in Figure 2-2. Published wrist stiffness studies typically neglect to differentiate between stiffness measured from flexion to extension and from extension to flexion (or from RD to UD and from UD to RD); so, it is not known what difference, if any, there is in 
the passive stiffness experienced by the wrist as a result of hysteresis. It is beyond the scope of this review to explore the origin and effects of this phenomenon in depth. The reader is referred to Gillard et al. (Gillard et al., 2000) for an examination of the effect on active and stimulated wrist response.

\subsection{Stretch Velocity}

Wrist joint biomechanics exhibit damping effects in addition to stiffness effects. Depending on the sophistication of the model being used to characterize wrist joint dynamics, the damping effects may be incorrectly included in calculations of the stiffness. One method for separating the stiffness from the damping is by conducting static or pseudo-static tests. In static tests, the wrist joint is perturbed to the desired location and the applied torque is measured during a period of zero velocity. The drawbacks of this method include its ability to record the applied torque only at the end-point of the test range — or throughout the test range if frequent stops are made — and its susceptibility to stress relaxation bias (see following section).

Pseudo-static tests are performed at a relatively low displacement velocity so as to minimize the damping effect. This method has the advantage of being able to record the applied torque throughout the entire range of the test motion. Formica et al. (Formica et al., 2012) reported that passive wrist stiffness estimates did not vary significantly with test velocity when varied from 0.1 to $0.2 \mathrm{rad} / \mathrm{s}$ (Formica et al., 2012). The authors have conducted similar tests after the fashion of Formica to determine the effect of strain-rate on passive wrist stiffness for flexion movements over velocities from $0.04 \mathrm{rad} / \mathrm{s}$ to $0.80 \mathrm{rad} / \mathrm{s}$. The results displayed a marked correlation between velocity and stiffness, the disparity between estimates being as large as $450 \%$. In general, the stiffness estimates were substantially equivalent for test velocities between 0.04 and $0.2 \mathrm{rad} / \mathrm{s}$. 


\subsection{Stress Relaxation}

The phenomenon known as stress relaxation is commonly observed during static or semi-static experiments. It manifests itself when the subject's wrist is perturbed to a predetermined nonneutral orientation and held there for a period of time. With the passing of time, the torque required to hold the joint at the specified orientation decreases. This behavior of the wrist has not been the subject of rigorous investigation.

\subsection{Exercise}

The influence of strenuous exercise prior to passive wrist joint stiffness measurements has not been adequately examined in the literature. We present here the results of several studies; the first two dealt with passive muscle stiffness in humans and felines, and the third pertained in a limited manner to the passive post-exercise stiffness of the human wrist joint.

Whitehead et al. (Whitehead et al., 2001) investigated the effect of exercise on active and passive muscle stiffness of the triceps surae in humans. The subjects' passive muscle stiffness was measured before and immediately after a period of exercise with daily measurements occurring over the next four days. The study was divided into two groups — an eccentric exercise group and a concentric exercise group. Only one of the patients' legs was exercised; the other was used as a control. The muscle stiffness in the concentrically exercised leg did not differ significantly from the control leg at any time during the 96 hours following the exercise session. The muscle stiffness in the eccentrically exercised leg increased immediately following exercise by $40.8 \% \pm 13.0 \%$, continued to rise till 24 hours post-exercise, and remained elevated by more than $20 \%$ throughout the 96 hours post-exercise. A study conducted on the feline medial gastrocnemius muscle showed that passive stiffness increased immediately following eccentric 
exercise to $142 \%$ of the pre-exercise value, and continued to rise to $191 \%$ of the pre-exercise value 40 minutes following the study (Whitehead et al., 2003).

Leger and Milner (Leger and Milner, 2000) conducted a similar study on the human wrist. Subjects' dominant wrists were conditioned using concentric and eccentric exercises until exhaustion of the wrist extensor muscles. One day after the exercise session, the passive wrist flexion $\mathrm{x}-\mathrm{ROM}$ had decreased by $15 \%$ from $82.8^{\circ}$ to $70.2^{\circ}$. The trend continued two days after the study, but increased steadily between day two and day ten to pre-exercise values. Passive wrist stiffness taken at a single neutral joint position, however, did not change significantly. It is unclear how large a region at the neutral position was included in the passive stiffness measurement.

It is unclear whether exercise significantly influences the passive stiffness of the wrist joint. Both studies by Whitehead - in the human triceps surae (Whitehead et al., 2003) and the feline medial gastrocnemius (Whitehead et al., 2001) — revealed that eccentric exercise results in the highly significant increase of passive muscle stiffness. Leger and Milner, working with the human wrist joint, reported a significant decrease in $\mathrm{x}-\mathrm{ROM}$, which is inextricably linked to passive joint stiffness, yet registered no significant change in passive joint stiffness when taken at the neutral position. It is left to the individual researcher to decide whether this single-point evaluation of post-exercise stiffness is a sufficiently adequate characterization of the phenomenon to reject the application of Whitehead et al. (Whitehead et al., 2003; Whitehead et al., 2001) to the wrist joint.

\subsection{Muscle Activation}

Muscle activation has the effect of increasing joint stiffness. Though it is beyond the scope of our present discussion, a brief review of the literature shows that muscle activation increases 
joint stiffness from passive levels of between 0.32 to $3 \mathrm{Nm} / \mathrm{rad}$ (De Serres and Milner, 1991; Gielen and Houk, 1984) to active levels of between 3.8 and $16 \mathrm{Nm} / \mathrm{rad}$ (Hogan, 1985; Leger and Milner, 2000; Milner and Cloutier, 1993). While measurements of passive wrist stiffness nominally occur in the absence of muscle activation, experimenters must recognize the nontrivial impact of improperly controlling for accidental muscle activation, which may significantly skew the results.

\subsection{Conclusion}

The wrist plays an important role in accomplishing daily tasks. The wrist's ability to perform its proper function may be affected by accident, repetitive-stress-injury, or other disorder, resulting in a decreased quality of life for those afflicted. Understanding wrist joint dynamics, the interdependence of the wrist joint with the other joints in the arm, and the process of neural planning that involves the wrist and arm to position the hand requires the explicit, detailed characterization of the passive stiffness encountered by the wrist.

Volumes of data have been collected and studied to understand and describe the behavior of passive wrist stiffness and the many factors upon which it depends. By taking a collective view of the results, we learn that passive wrist stiffness depends significantly on methodological parameters such as the range-of-motion tested, the definition of the neutral position, and movement constraints, as well as physiological factors such as age, gender, orientation of the arm, direction of movement, strain-rate, repetition of movement, stress relaxation, and preexercise. These findings lay the foundation for future work and indicate the specific areas which must be controlled both before and during data acquisition.

Not surprisingly, there are considerable differences in the reported literature that stand in need of resolution. It is still unclear whether pre-exercise increases passive wrist stiffness-as it 
does passive muscle stiffness (Whitehead et al., 2003; Whitehead et al., 2001) — or whether it has no discernible affect at all (Leger and Milner, 2000). The test velocity (or strain rate) used during data acquisition reportedly has little effect on passive stiffness if its variance is limited to a certain range (Formica et al., 2012), but the authors have seen that increasing the bounds of that range has a significant effect on passive stiffness. The most critical discrepancy in literature, however, comes from the widely varying reports of the values of passive stiffness in the joint, with some reports differing by $1500 \%$.

In addition to the discrepancies, there are several areas of research that have been investigated in relatively few papers and other areas that have never been the subject of scholarly investigation. Johns and Wright (Johns and Wright, 1962) conducted a study to determine the relative contributions of the joint capsule, tendons, muscles, and skin to passive joint stiffness in the feline wrist, applying their relative contributions to the human metacarpophalangeal joint. A similar study could be done on the human wrist joint to determine the relative contributions of the various tissues surrounding the joint to the overall stiffness observed. The correlation between age and passive wrist joint stiffness has not been directly investigated in the literature. Likewise, the stress relaxation phenomenon lacks sufficient explanation.

The groundwork that has been laid over the past several decades of research better prepares future investigators to properly account for and fully characterize the various factors influencing passive wrist stiffness. As new measurements and characterizations are made, they will be invaluable to the fields of neurophysiology, biomechanics, preventative care, and rehabilitative care. 


\section{METHODOLOGY}

Though our technique for passive wrist stiffness characterization built upon the substantial work of others who preceded us, several important aspects were either new and had to be investigated thoroughly before testing could begin or were known phenomena that had not been sufficiently characterized by previous studies such that their exact effects could be determined without additional research. This chapter presents the work that was accomplished before we could begin gathering data from subjects. It also includes an in-depth treatment of the post-processing techniques that were used to convert the raw torque-position data recorded by the wrist rehabilitation robot into the stiffness matrices that were our ultimate goal. As described in previous chapters, the three degrees-of-freedom (DOF) of the wrist joint and forearm are wrist flexion-extension (FE), wrist radio-ulnar deviation (RUD), and forearm pronation-supination (PS).

\subsection{Preparatory Work}

This section explores the body of research that was completed in preparation for the main experimental study. It progresses chronologically through the work that was done.

\subsubsection{Preliminary Stiffness Studies}

As has been noted in previous chapters, the reported values for passive wrist stiffness found in literature before we began this study spanned an enormous range several orders of magnitude in 
breadth. The fact that reports have been so widely disparate reflects the extreme impact of measurement technique on results and highlights the need to verify our own results with separate measurement techniques that avail themselves of distinct methods.

The chosen method for our main study was based on work that had been developed at MIT. Researchers there designed and constructed the prototypes for a wrist rehabilitation robot that was eventually spun-off into its own company, Interactive Motion Technologies (IMT). The robot that we used for data collection was manufactured by IMT. We will discuss the particulars of the robot, its capabilities, and use further on in this chapter.

The results of a two DOF study that utilized the IMT wrist robot to characterize stiffness in the region $\pm 15^{\circ}$ in the FE-RUD plane were available when we began to conduct our research (Formica et al., 2012). Because the 3DOF nature of our study added new factors with unknown effects to the experiment, we chose to maintain the same methods as the previous study 1) where we determined that the previous researchers had used the best technique or 2) where alterations would have compounded effects in such a way as to have prevented delineating between causes and their effects during post-processing. To illustrate the first case, we maintained the same parameters for the internal $\mathrm{P}+\mathrm{D}$ control of the robot and test velocity after determining from our own experiments that they were sufficiently appropriate for the study. To illustrate the second case, we used the same range of motion as the previous study $\left( \pm 15^{\circ}\right)$ because it had been shown to be linear and because it would have been impossible in post-processing to determine whether significant new findings were a result of the increased ROM or a result of the study having been performed in 3DOF.

Returning to the original line of discussion, the high degree of variation observed in reported values for passive wrist stiffness presented a great cause of concern. The passive 
stiffness of the general population did not change between studies, but the methodologies of measuring did. If our results were similar to those reported by the group at MIT, it would mean that the process was repeatable and not necessarily that the numbers themselves were accurate. If, on the other hand, the numbers we arrived at were distinct from those obtained at MIT, what guarantee would there be that the new numbers were more accurate than the old numbers. The only argument would be a verbal justification and not a numerically derived justification. We felt that before conducting a full experiment on subjects, we needed to verify by some means other than the IMT robot that the data we were collecting with it was correct.

To this end, we devised several low-tech preliminary studies to estimate passive wrist stiffness via several distinct methods, relying on distinct measurement techniques. These preliminary studies, we hoped, would help to delineate a "ballpark" range of estimates within which the actual passive stiffness of the wrist would be found to reside. In the end, we measured passive stiffness on several subjects using the following techniques.

1. Gravity: The subject's forearm were supported on a table top and their wrist was allowed to hang freely under the influence of gravity. The angular deflection of the second metacarpal with respect to the long axis of the forearm was measured using a hand-held goniometer. Angular deflection due to gravity was measured in flexion and extension, repeated for both right and left hands. The weight of the subjects' hand was calculated using de Leva's (de Leva, 1996) modified tables, the joint torque was found using the weight of the hand and approximate distance between the center of mass of the subjects hand and the wrist joint (also calculated using de Leva's tables), and the stiffness of the joint found as the ratio of joint-space torque over deflection. 
2. Pendulum, clamp, and linear force transducer (LFT): A custom made clamp was fashioned to restrain the motion of the subject's arm by affixing to the wrist. The mechanism operated by "sandwiching" the subject's wrist between a stationary upright and a moveable upright that translated on a screw-mechanism. The two uprights pressed against the dorsal and ventral aspects of the distal protuberances of the radial and ulnar heads. The subject's wrist being thus affixed, the weight of the hand was supported by a long pendulum suspended from a point high above the subject that allowed for displacement of the wrist in flexion and extension without inducing significant radio-ulnar deviation. A linear force transducer attached to the pendulum and was used to measure the force required to displace the hand in flexion by $5^{\circ}, 10^{\circ}$, and $15^{\circ}$.

3. Pendulum, no clamp, and LFT: Because the effect on passive stiffness of compressing the tendons across the carpal tunnel has not been reported in literature, we repeated the pendulum study without the use of the dorsal-ventral clamp. Movement of the subject's wrist was instead restricted by the researcher placing one hand on the subject's dorsal end of the radius (bone) and pressing downward. In this manner, movement could be restricted without applying pressure directly over the wrist tendons.

4. Peg-board and goniometer: The initial experiment, Goniometer and gravity, was then redone with more careful attention to the attitude of the wrist as it deflected under the influence of gravity. A peg-board (such as may be found in a typical workshop) was used to provide horizontal supports upon which the wrist was allowed to rest during the study. Again, the deflection of the subject's wrist was measured with a goniometer, and the stiffness was calculated as above. The process was repeated for ulnar deviation. 
5. Peg-board and LFT: Finally, we combined the LFT tests with the peg-board test. The horizontal supports were again used to position the subject's wrist. A sling was placed under the subject's hand and attached to the LFT that permitted the force to be measured as the subject's hand was displaced. Starting from a position of flexion (caused by gravity), the subject's wrist was raised back into the neutral position (with the second metacarpal aligned with the long axis of the forearm) and the required force was measured. The stiffness was calculated as the force required to affect the displacement divided by the angular difference between starting position and final position. During the experiment, it was discovered that the stress relaxation in the wrist joint caused the sustaining force to decrease as time progressed. We therefore recorded the force at two time intervals: first, at the moment when the wrist joint first re-aligned with the neutral position, and second, after a period of about 30 seconds following re-alignment.

The results of the preliminary studies as well as the results reported in several published research articles are presented in the table below (Figure 3-1). The range of values gathered during the preliminary studies lines up favorably with the values from literature. In some cases, the numerical values are similar even though the ROM over which they were obtained are significantly different; however, this may be accounted for by the large linear region of stiffness which has been demonstrated to exist throughout much of the wrist's ROM. As a result of the preliminary studies and their strong correlation to established values, we were confident that the actual passive stiffness of the wrist would ultimately fall within the identified region.

\subsubsection{Re-programming the Robot}

Before we could use the robot to conduct our studies, it had to be reprogrammed. The IMT wrist rehabilitation robot is marketed primarily to rehabilitation clinics throughout the world. It comes with pre-programmed exercises, evaluations, and other tasks designed to assist in rehabilitation 
of patients with movement disorders. This works fine in a clinical setting where customization is not necessary, but the complex experimental protocols that we desired to run required a much more flexible and customizable layout.

\begin{tabular}{|l|c|c|c|}
\cline { 2 - 4 } \multicolumn{1}{c|}{} & FE/FE & RUD/RUD & RUD/FE \\
\hline (Rijnveld and Krebs, 2007) & -- & 1.45 & -- \\
\hline Peg board w/LFT & 0.12 & -- & -- \\
\hline Peg board w/goniometer & 0.14 & 1.42 & -- \\
\hline (Lehman and Calhoun, 1990) & $0.002-0.15$ & -- & -- \\
\hline Gravity & 0.31 & -- & -- \\
\hline (Axelson and Hagbarth, 2001) & 0.34 & -- & -- \\
\hline (Gielen and Houk, 1984) & $0.32-0.70$ & -- & -- \\
\hline (Formica et al., 2012) & 1.19 & 1.67 & -0.175 \\
\hline Pendulum w/clamp & 1.48 & -- & -- \\
\hline Pendulum w/o clamp & 1.57 & -- & -- \\
\hline (Leger and Milner, 2000) & 2.2 & -- & -- \\
\hline (De Serres and Milner, 1991) & 3.0 & -- & -- \\
\hline (van Eesbeek et al., 2010) & 3.05 & -- & -- \\
\hline
\end{tabular}

Figure 3-1: Comparison of passive wrist stiffness measurements from preliminary studies (black) to reports in literature (gray).

A full summer was spent first deciphering the source code for the default programs and then writing completely new programs to handle the same processes with greater flexibility. This included a drastic rewrite of the main program including many alterations to the sublevel programs. For example, the $\mathrm{P}+\mathrm{D}$ controller that plans robot movement in the FE-RUD plane was coupled. That is to say, it was not possible to control FE and RUD separately. This presented a significant problem to our experimental design; so, two new separate controllers had to be written to allow for control of FE or RUD independently of the other. The most important modification allows for researchers to have full control over the location and repetition of endpoints for executing movements. This is accomplished by means of elaborate vectors of points 
generate by MATLAB code written expressly for the purpose. The result of these modifications is to allow researchers to design precise, customizable experiments.

\subsubsection{Determination of Robot Parameters}

Having identified the most plausible region of passive wrist stiffness and having modified the robot to accept customized commands, we proceeded to conduct a series of experiments designed to identify the most favorable parameters at which to set the robot during the main study. As noted, the device used during principal data gathering was a three DOF wrist rehabilitation robot from Interactive Motion Technologies. In layman's terms, the robot is a suped-up, motorized joy-stick. The robot consists of a manipulandum capable of both sensing and actuating movement of the wrist joint in FE and RUD. The subject's hand interfaces with the robot by a vertical handle that comes standard on the robot. The manipulandum rides on an arcshaped carriage that senses and actuates movement of the forearm in PS. The maximum jointspace torque in any of the three DOF is about $2 \mathrm{Nm}$.

The robot employs a straight-forward $\mathrm{P}+\mathrm{D}$ controller for movement; both the proportional and derivative gains may be set by the experimenter. A previous study utilizing the wrist robot in two DOF had reported values of 10 and 0.1 as the $\mathrm{P}+\mathrm{D}$ gains. We determined to verify those results on our own machine, optimizing the gains for smooth movements with minimal resonance.

Parameter crossing (PC) tests were conducted by running the empty robot through the area from neutral to 0.4 radians of flexion, varying the proportional gain through the vector $[10$, $15,20,25]$ and the derivative gain through the vector $[0.1,0.2,0.3,0.4]$. Each movement was repeated five times in succession and the runs were averaged together. It was determined by 
visual inspection of the torque-position plots that $\mathrm{P}+\mathrm{D}$ gains of 10 and 0.1 would result in the smoothest movements.

These optimal parameters were then used as the basis for a follow-up comparison of two separate smoothness conditions: 1) using calculated integral minimum jerk profiles (IMJ), and 2) using IMJ and derivative integral minimum jerk profiles (DIMJ). The best runs from the PC, IMJ, and IMJ+DIMJ tests are presented graphically below (Figure 3-2). We concluded that the best combination offering low resonance and stability was the 100_10_0.1 (IMJ) test. Deciphering the naming convention yields the following parameters: single-sided time $100 \mathrm{~ms}$ ( $0.8 \mathrm{rad} / \mathrm{s}$ test velocity), proportional gain 10 , and derivative gain 0.1 , with integral minimum jerk profiles.

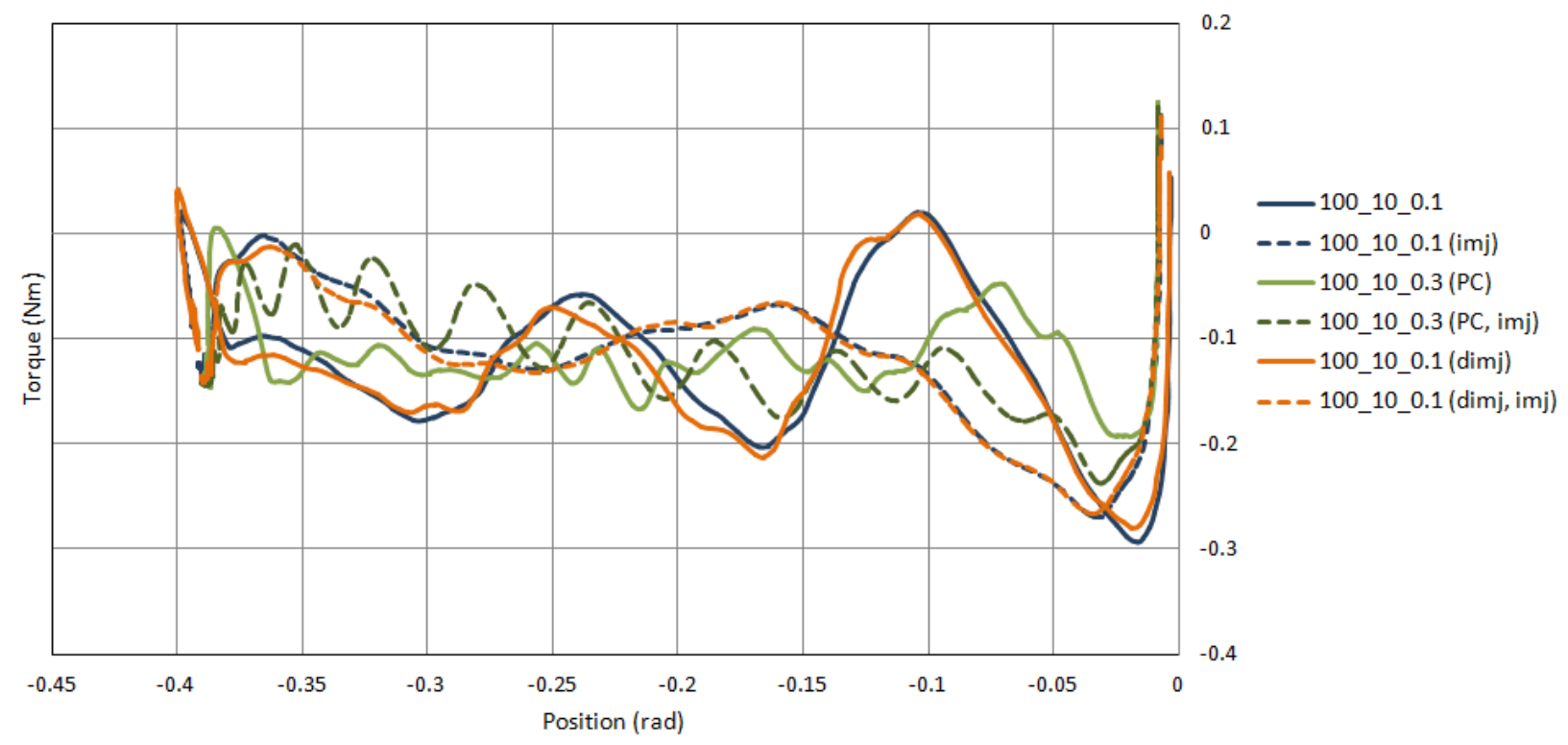

Figure 3-2: Comparison of torque-position to determine the smoothest operating parameters. Studies performed with empty robot. PC—parameter crossing tests, IMJ-integral minimum jerk profile, DIMJ-derivative integral minimum jerk profile.

This new optimal set of test parameters (10_0.1_imj) was used as the base for an additional follow-up study using a human subject. Tests were conducted by running the robot 
with a single, right-handed subject (tests on right wrist). Again, the test region included the area from neutral to 0.4 radians of flexion. For these tests, the average velocity was varied through the vector $[0.80,0.40,0.27,0.20,0.16,0.13,0.11,0.10,0.05$, and $0.04 \mathrm{rad} / \mathrm{s}]$.

The purpose of this study was to determine the effect of test velocity on passive stiffness estimates. As test velocity increases, the contributions of reflex action in the wrist muscles, viscoelastic damping, and the inertia of the hand increase as well. There is also the possibility that additional, undocumented effects alter the passive stiffness of the wrist as velocity varies. Each test was repeated five times in succession. The first repetition of each test was discarded due to thixotropy, while the remaining repetitions were averaged together (Figure 3-3). The short-range-stiffness and torque-position end effects were also discarded, and a linear stiffness estimate for the midrange was calculated (Figure 3-4).

The velocity tests were repeated over four separate days to investigate the secondary effect of passive stiffness naturally varying from day to day. The linear midrange stiffness (MRS) estimates from all of these speed tests (ST) are presented below (Figure 3-4) along with a linear curve fit of each full test. By extrapolating the linear curve fits (dashed lines) towards a test velocity of $0.00 \mathrm{rad} / \mathrm{s}$, we may infer that a static test of passive wrist stiffness would result in a measurement between 0.8 and $1.0 \mathrm{Nm} / \mathrm{rad}$ for flexion. It is interesting and important to note that estimates changed by as much as $300 \%$ for the same velocity on different days-e.g. ST-I, ST-III, and ST-IV at a velocity of $0.80 \mathrm{rad} / \mathrm{s}$ (Figure 3-5). 
Figure 3-3: Torque-position plots for a typical experimental run. Note how the first repetition exhibits thixotropic effects, which quickly die out, leading the following four repetitions to be essentially identical. This particular test was performed using a test velocity of $0.80 \mathrm{rad} / \mathrm{s}$.

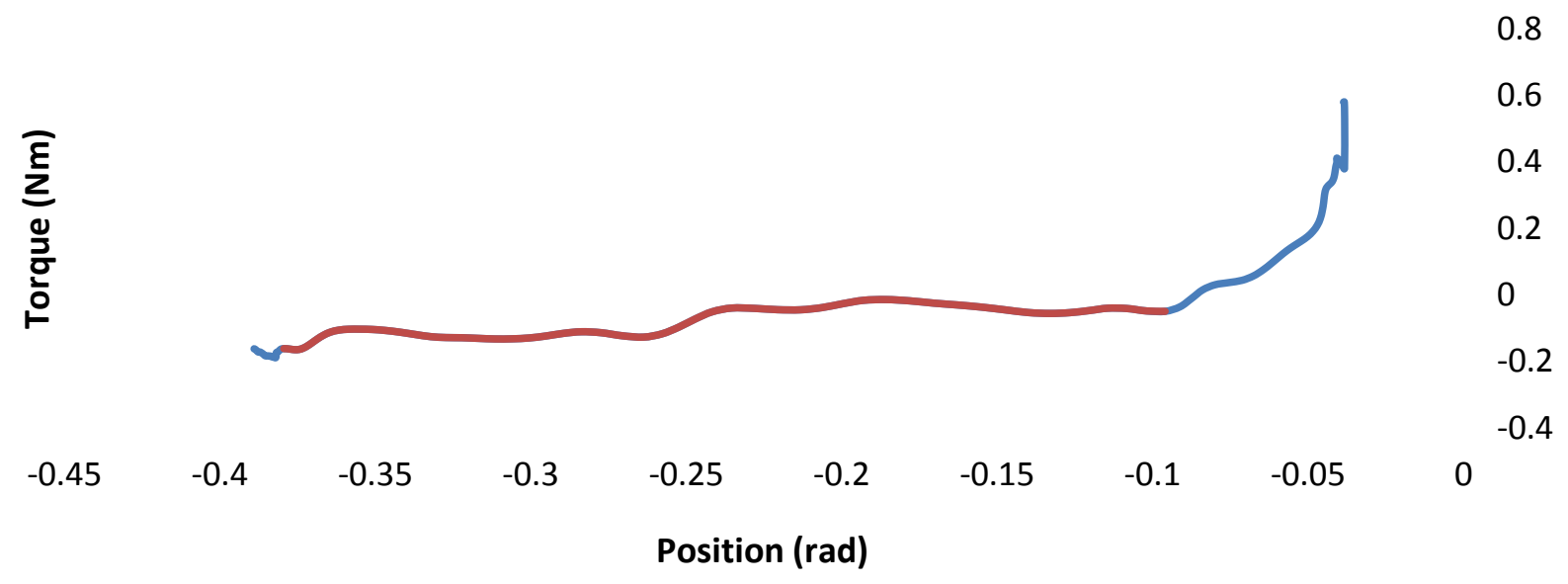

Figure 3-4: Average of R2-R5 for the velocity test presented in Figure 3-3. The short-range-stiffness observed in the initial region (from -0.05 to $\mathbf{- 0 . 1}$ rad) was not included in the linear stiffness calculation. The end effects (near $\mathbf{- 0 . 4} \mathbf{~ r a d}$ ) were also excluded from the linear estimate. 


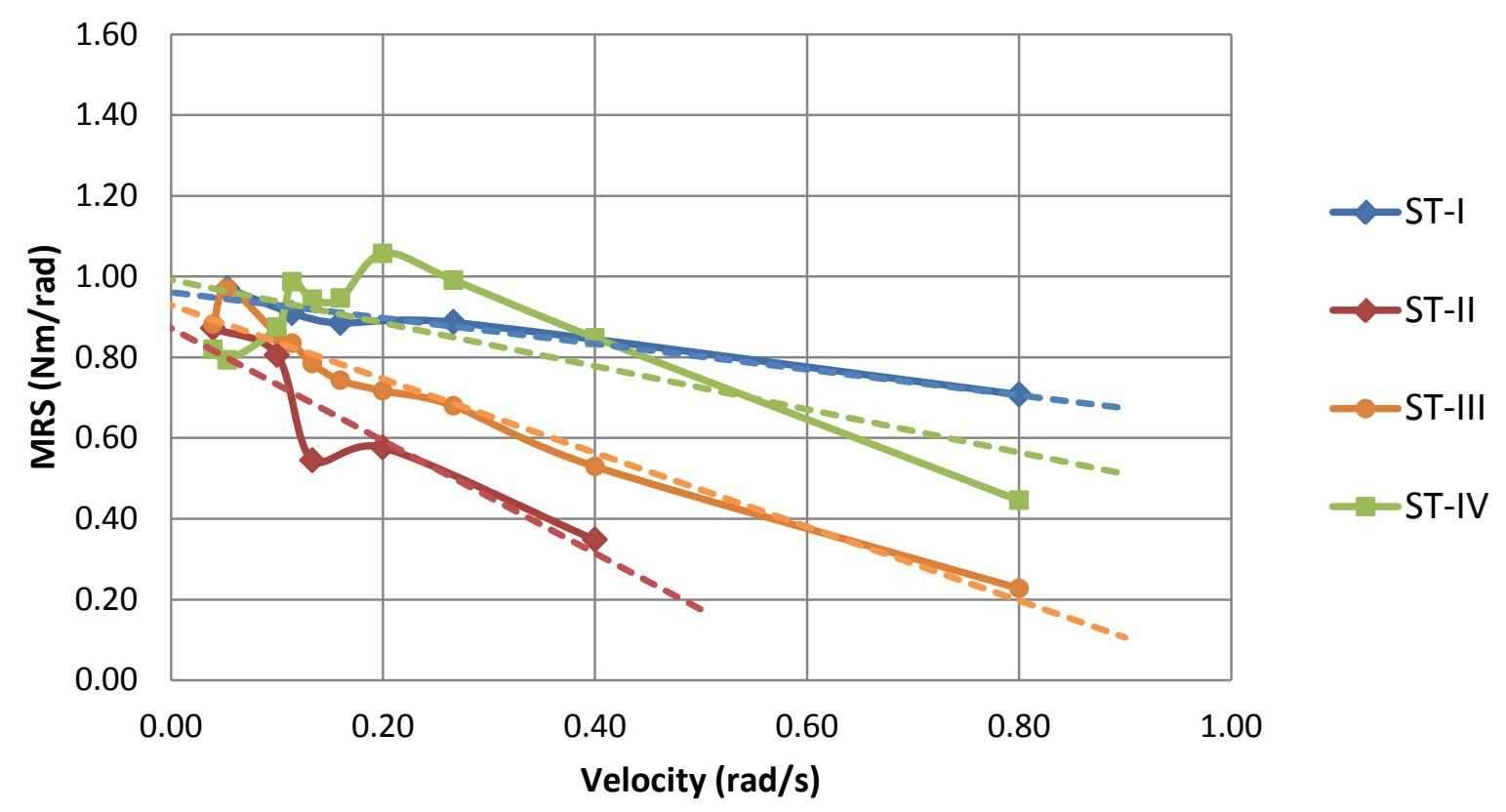

Figure 3-5: Midrange stiffness (MRS) estimate for flexion from 0.0 to 0.40 radians for varying test velocities. Each color grouping was performed on a different day. All tests for a single subject.

\subsubsection{Robot-Human Interface}

The IMT wrist rehabilitation robot comes with a default vertical handle configuration. Like the default programs, this is sufficient for clinical use but it may significantly affect passive stiffness measurements. The work of Gehrmann et al. (Gehrmann et al., 2008) shows that changing the orientation and freedom-of-movement of the fingers can alter wrist range-of-motion (ROM) by as much as $30 \%$. It is reasonable to infer that similar increases in passive wrist stiffness are the real reason for the reduction in ROM. If one is studying the passive stiffness associated with activities-of-daily-living (ADL) or occupational tasks that require the hand to conform to a handle, then the default vertical handle configuration should be used. If, however, a general characterization of passive wrist stiffness without regard to specific ADL or occupational tasks, then a different robot-hand interface must be designed to permit the fingers to move freely during the experiment. 
The device shown in Figure 3-6 was designed to meet these requirements. It straps to the patient's hand at the distal end of the metacarpals and does not restrict the free movement of the fingers. This robot-hand interface was used in all principal testing.

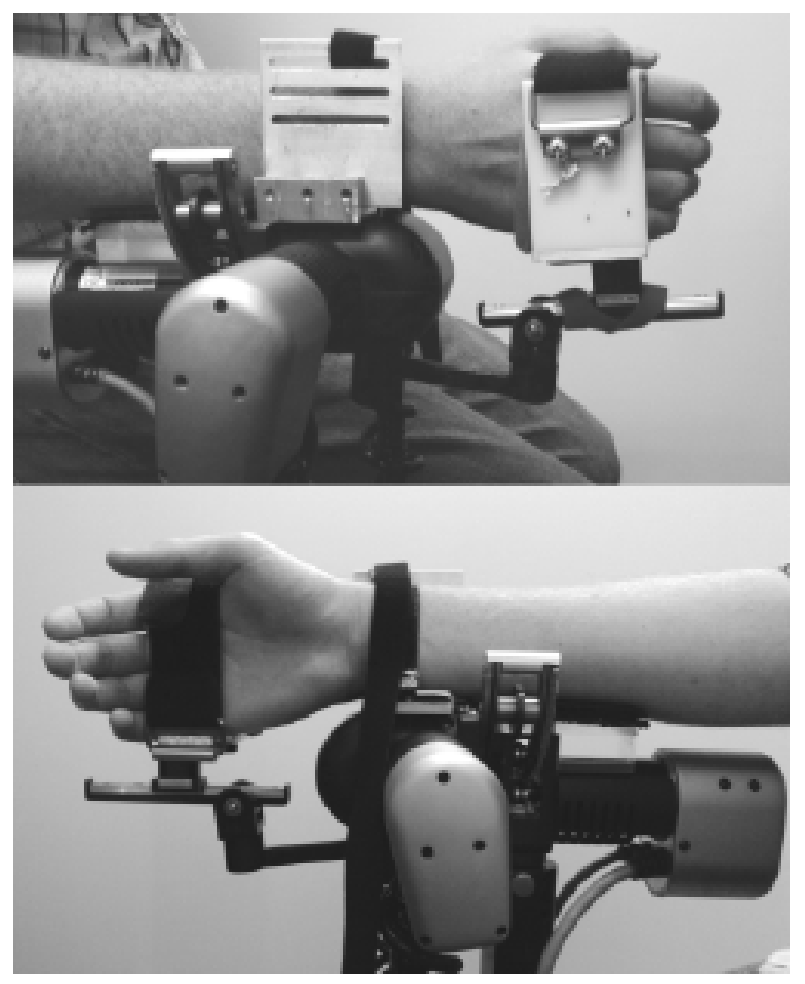

Figure 3-6: Custom-built robot-hand interface. The device attaches to the distal metacarpals and permits free movement of the fingers during the experiment.

\subsubsection{Point Distribution}

The chosen ROM for characterization is $\pm 15^{\circ}$ in FE, RUD, and PS. While it is easy to specify the range, the selection of actual target values for [PS, FE, RUD] within that range is non-trivial. There are two basic methods for uniformly distributing points on a sphere. For the present discussion, the azimuth angle theta $\theta$ lies in the xy plane (FE-RUD plane); the elevation angle phi $\varphi$ is formed by the xy plane and the $\mathrm{z}$ axis (PS axis). 
In the first method, points are uniformly distributed in theta from 0 to $2 \pi$ and in phi from 0 to $\pi$. Theta and phi are then converted from spherical coordinates to Cartesian coordinates. This method is straight forward, however, a linear distribution of points along phi and theta does not account for the curvature of the spherical surface. As a result, the extremes of phi are oversampled and the middle is undersampled.

In the second method, the calculation of phi is modified to account for the curvature of the spherical surface and compensate for oversampling and undersampling. A parameter $u$ is divided into $N$ divisions from 0 to 1 . Phi is calculated as $\operatorname{acos}(2 * u-1)$. When viewed in the FE-RUD plane, the second method creates a large gap between the center of the cluster and first ring of points. The first method was used in the present study because it's FE-RUD projection does not create such a gap (Figure 3-7 and Figure 3-8).
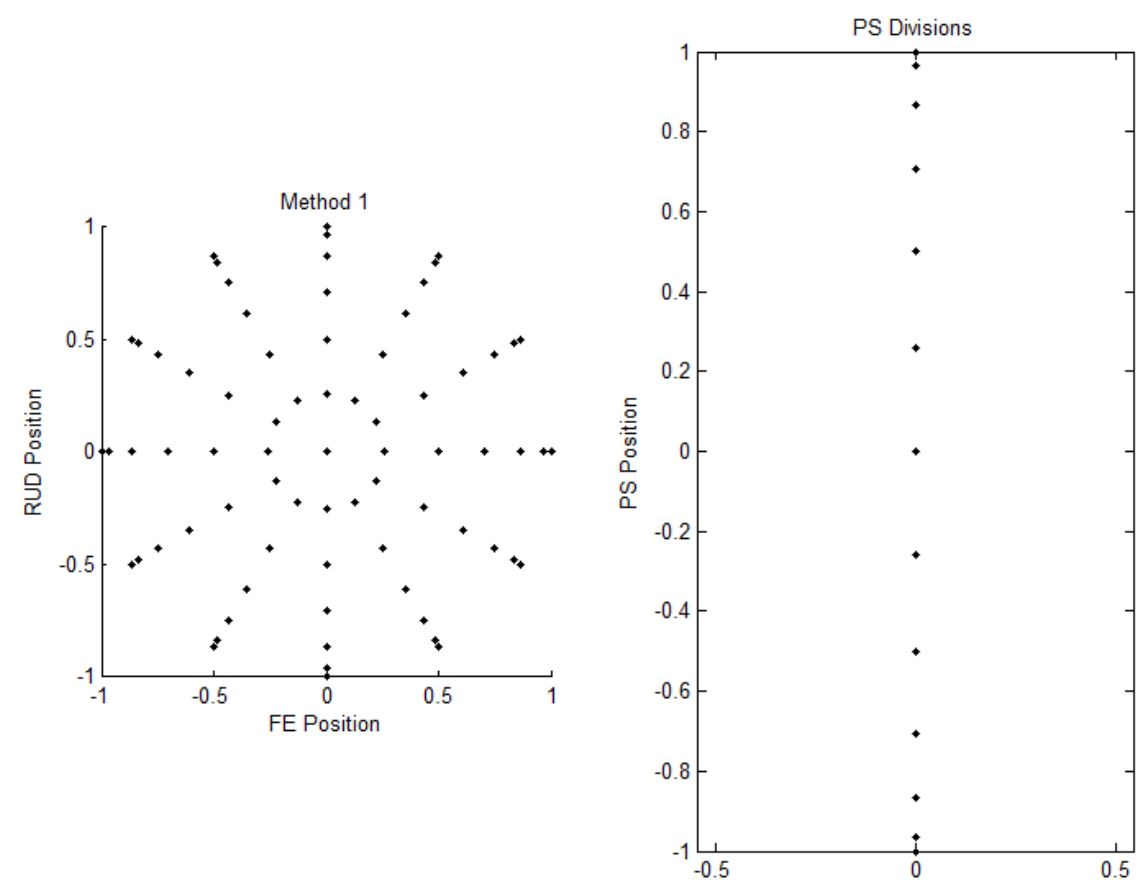

Figure 3-7: Uniform distribution of points on a sphere by Method 1, resulting in a non-uniform distribution of points in the PS axis. 
The resulting distribution contained 134 distinct points in [PS,FE,RUD] space. The FERUD plane was divided into 12 segments $30^{\circ}$ apart. The PS axis was divided into 12 segments $2.5^{\circ}$ apart.
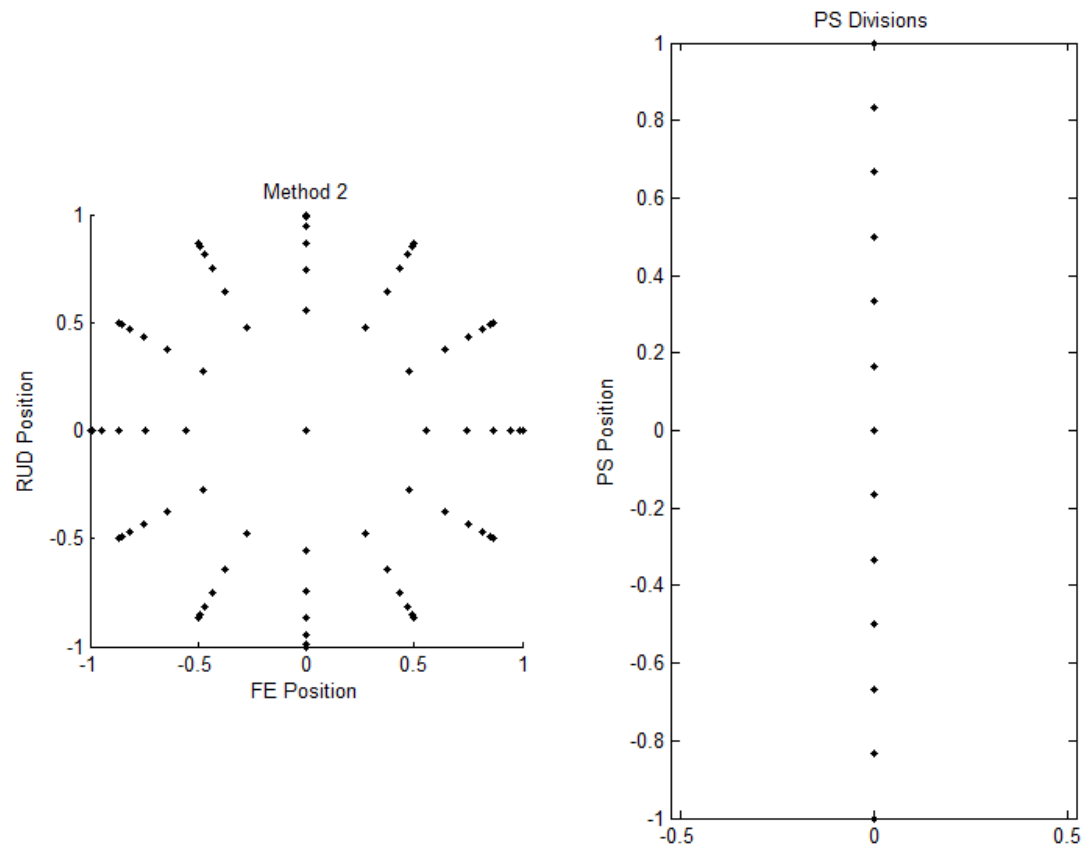

Figure 3-8: Uniform distribution of points on a sphere by Method 2, resulting in a uniform distribution of point in the PS axis.

\subsection{Experimental Procedure}

Following the all preliminary studies and preparations, we were ready to conduct the central experiment related to this research: measuring the torque created by passive wrist stiffness throughout the desired ROM.

\subsubsection{Subject Population}

The study population consisted of ten right-handed subjects (five female) whose mean age was 24 years old. The youngest subject tested was 18 years old, while the oldest subject was 38 years 
old. None of the subjects had a history of neurological or biomechanical disorders affecting the wrist and forearm. All of the subjects were instructed not to participate in heavy exertion of the upper body in the 48 hour period preceding the study to reduce the likelihood of measuring passive stiffness during the four day period of elevated passive stiffness that follows eccentric exercise. Each subject gave informed consent consistent with the procedures outlined by BYU's Institutional Review Board.

\subsubsection{Calibration Marks}

A series of marks were made on the subject's hand, wrist, and forearm to assist in anthropometric measurements and calibration in the wrist robot. The marks are subdivided into three distinct groups: Group 1) Co-linear marks used for calibration along the dorsal side of the hand, wrist and forearm. Group 2) Co-linear marks used for calibration along the lateral side of the hand, wrist, and forearm-i.e. along the second metacarpal and the radius of the forearm. Group 3). A pair of marks on the dorsal and ventral sides of the hand for calculating moment arms when using the hand-held dynamometer that accompanied maximum voluntary contraction (MVC) measurements. There exact definitions of each group of marks are presented here for convenience. In all cases, the final mark should be made with the subject's limbs oriented as close to the calibration position as possible: significant skin artifacts may result if the mark is made while the arm is not oriented in the calibration position.

\section{Group 1}

- Elbow joint center (EJC): Located on the lateral epicondyle of the humerus. Find by placing the thumb on the lateral epicondyle of the humerus and observing the motion of the elbow as it is flexed and extended. Mark the EJC as nearly to the center of rotation on the lateral epicondyle as possible. 
- Dorsal wrist joint center (D-WJC): First, start on the distal end of the third metacarpal and follow it proximally until the end of the bone is reached. Place a mark just as the bone rounds off. Next find the distal end of the dorsal aspect of the radial head in the middle of the wrist (with respect to lateral-medial). Mark the spot where the radial head rounds off. Finally, mark the midpoint between the two marks just made: this is the location of the D-WJC proximal-distally. To find the lateral-medial location of the D-WJC, have the subject position their hand with the thumb vertical. Mark the midpoint of the dorsal aspect of the wrist in the plane of the proximal-distal D-WJC mark.

- Third metacarpophalangeal joint center (MCP3): The EJC, D-WJC, and MCP3 must all be aligned during the study, however, the robot-hand interface frequently obscures any marks made on the MCP3. To alleviate the problem, mark the center (dorsal) of the third metacarpophalangeal joint. Next, mark the center (dorsal) of the proximal interphalangeal joint on the third phalange (PIP3). Finally, connect the two joint centers with a straight line. This line will be used to align MCP3 (likely obscured) with the D-WJC and the EJC during calibration.

\section{Group 2}

- Second Metacarpophalangeal joint center (MCP2): Mark the midpoint (lateral) of the second metacarpophalangeal joint. Keep in mind that this mark will be used to align MET2 and the radius during calibration.

- Lateral wrist joint center (L-WJC): With the subject's forearm horizontal and thumb vertical, stand above the wrist joint and look straight down. Mark the 
center of the wrist (ventral-dorsal) at the same level as the previous D-WJC marks.

- Elbow trough (ET): Unfortunately, there is no objective, clinically practical way of defining the center of the elbow trough (or cubital fossa). With the subject's shoulder at $0^{\circ}$ abduction, forearm horizontal, thumb vertical, palpate the lateral and medial epicondyles of the elbow. Visually mark the center of the joint as well as possible using the epicondyles as a reference.

\section{Group 3}

- Palmar edge mark (PEM: Following the wrist robot portion of the experiment, maximum voluntary contraction (MVC) measurements will be taken. For future reference, we will be using a hand-held dynamometer to register the maximum force generated during the subject's MVC. Place the dynamometer on the subject's palm and make a distinct mark (it is suggested that you use a different colored pen than was used for Groups 1 and 2) at the proximal edge of the sensor's contact pad.

- Dorsal edge mark (DEM): Place the dynamometer in a comfortable location on the back of the subject's hand. Again, place a mark at the proximal edge of the sensor's contact pad.

\subsubsection{Anthropometry Measurements}

Anthropometric measurements were gathered using the calibration marks and saved in a patient data sheet similar to the one shown in Figure 3-9. These measurements were used in calculating the mass of each subject's hand using de Leva's tables (de Leva, 1996), the center of mass for each subject's hand, and the moment arm's used by the dynamometer. 


\subsubsection{EMG Placement}

One of the most crucial aspects of conducting a study on passive joint stiffness is maintaining and verifying the "passive" condition of the subject's muscles. As was explained in the literature review, activating the muscles surrounding the wrist joint drastically increased the stiffness of the joint. It was of primary importance to us that the subject's muscles were demonstrably dormant during the experiment. In all, six wireless EMG sensors (Delsys, MA) were used to monitor the primary muscles of the wrist joint, a primary pronator muscle, and a secondary supinator muscle.

Sensors were carefully placed on 1) the extensor carpi radialis longus and extensor carpi radialis brevis (grouped for convenience and referred to collectively as the ECR), 2) the flexor carpi radialis (FCR), 3) the extensor carpi ulnaris (ECU), 4) the flexor carpi ulnaris (FCU), 5) the pronator teres (PRO), and 6) the biceps bracii (BIC; the biceps is a secondary supinator; all primary supinators cannot be measured with surface EMG). Explanation of the exact technique is best achieved by demonstration; therefore, we will not provide a textual description.

\subsubsection{Robot Calibration}

Once the subject has been prepared with the calibration marks and EMG sensors, it is time to seat the subject at the robot and calibrate it to their specific anthropometry.

An ergonomic chair was used to seat subjects because it allowed for the subject to sit all the way up against the robot's frame without getting the subject's knees in the way of the robot's movements. The chair was situated so that the subject's shoulder was oriented at $0^{\circ}$ abduction (the importance of this was explained in the section on neutral position from Chapter 2). The subject's forearm rested on the robot's frame, which was adjusted to be at a comfortable height (determined by the shoulder's being level and by questioning the subject). The hand was 
strapped into the custom-built interface, and straps were attached across the subject's wrist, pulling the dorsal aspect of the wrist into contact with a vertical plate attached to the robot's PS carriage.

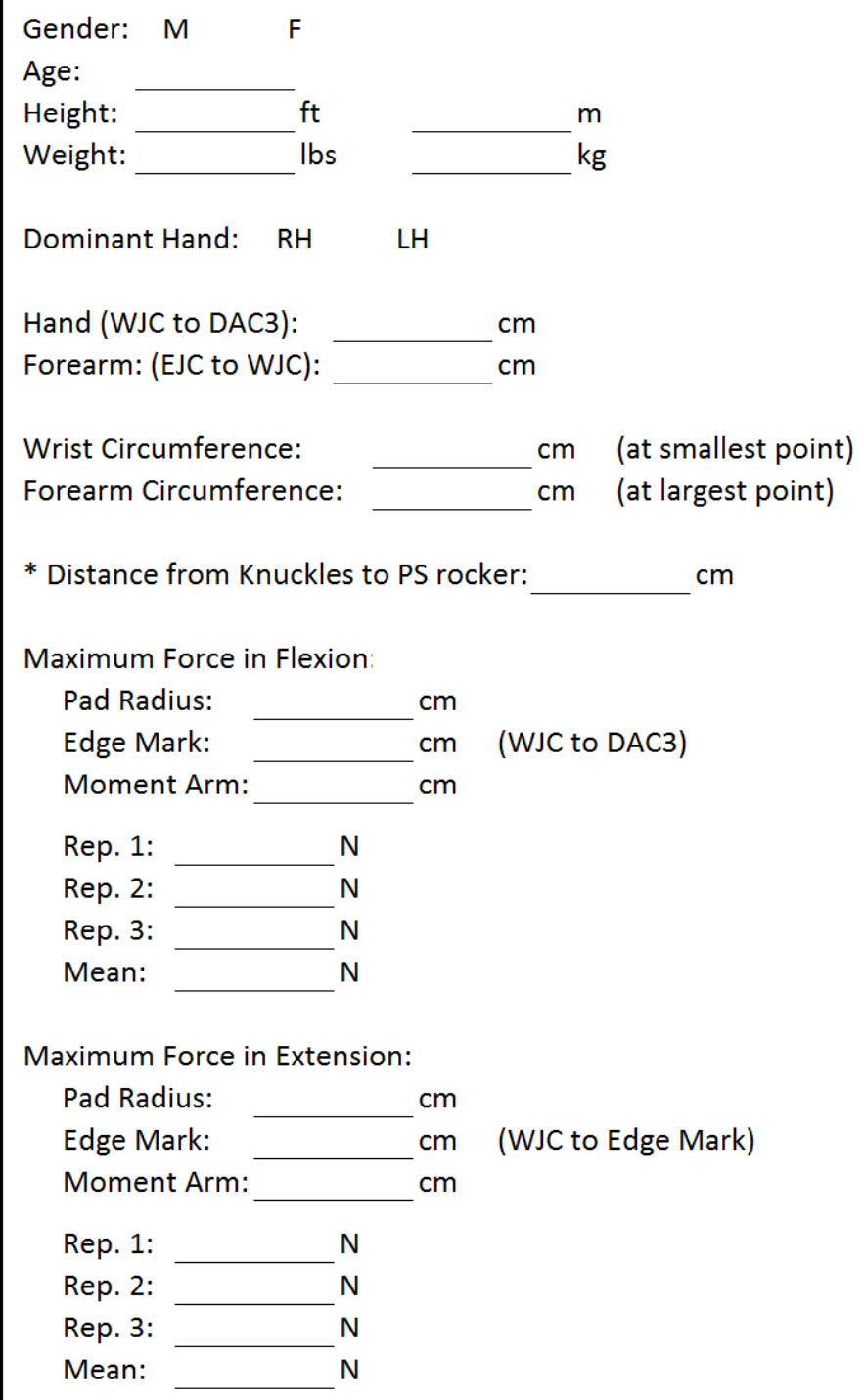

Figure 3-9: Anthropometric data sheet used in the study.

The calibration marks from Group 1 and Group 2 were used to calibrate the robot to the subject. A laser level was set up to intersect the EJC and WJC of the subject. The neutral position 
of the robot in RUD was then varied until the line connecting MCP3 and PIP3 was collinear with the laser level line.

Similarly, a straight edge was used to align the ET, L-WJC mark, and the MET2 mark from above. To accomplish this, the neutral position of the robot was varied in FE-RUD until the appropriate position was found. This new position, along with the position in RUD found when aligning Group 1, was hardcoded into the software for use during that subject's test.

This calibration ensured that the subject was in the proscribed neutral position at the start of the study. Upon completion of the calibration, the subject was instructed to remain relaxed and not to interfere with nor assist the robot during the study. The robot session lasted approximately 50 minutes.

\subsubsection{Maximum Voluntary Contraction}

Following the robot session, the subject was seated at a table and maximum voluntary contraction measurements were gathered using the EMG sensors and their accompanying software package. First, the dynamometer was placed in the subject's palm (carefully aligning it with the PEM mark made earlier. The researcher supported the person's arm at the wrist and applied pressure on the palm with the goniometer frequently coaching the subject to push as hard as possible. The coaching continued until the subject's maximum was reached and the researcher overpowered the subject through mechanical advantage. The process was repeated three times and the averaged result represented the flexion MVC. The process was repeatedly in a like manner using the DEM to gather the extension MVC.

Next, the pronation MVC was measured as the researcher grasped the subject's wrist firmly and, again coaching the subject verbally, applied a supinating torque until the subject was overpowered. The final MVC involved the biceps - a secondary supinator. This was 
accomplished by the researched placing one hand on the subject's shoulder and pulling out on the subject's wrist with the other hand until the subject was overcome.

In post-processing, the data from the MVC experiments was exported as a CSV file and imported into MATLAB.

\subsection{Post-Processing}

Following collection from the experiments, the data were processed using a custom written MATLAB program. The program read in all data files and EMG files, filtered them appropriately, identified EMG interference and removed the corresponding data files, performed multilinear regressions to obtain the $3 \times 3$ stiffness matrix, compensated the stiffness matrix for gravity and robot dynamics, performed statistical analysis on all of the results from each of the ten subjects, and created necessary charts and visualizations.

\subsubsection{Torque-Position Processing}

The IMT wrist robot output included position data and applied voltages on the motors. The voltages were converted into robot-space torques using the inverse of the motor equations found in the robot's source code. Once the robot-space torque had been found, both the torques and position data were converted from robot coordinates to standard ISB coordinates. Because of the short-range-stiffness effect, the first $2^{\circ}$ of movement were calculated for each distinct outbound or inbound motion and were removed. It was then possible to calculate the $3 \times 3$ stiffness matrix using multilinear regression. 


\subsubsection{EMG Processing}

The EMG data from both the robot and the MVC files were read into MATLAB and immediately detrended, rectified, and filter with a $2^{\text {nd }}$ order Butterworth filter set at a cutoff frequency of 3 Hertz. The maximum value from each repetition of the MVC data was found, and each of the three MVC measurements were averaged to give one mean MVC for each EMG sensor. The robot EMG files were then averaged across the whole movement (outbound or inbound). This average EMG reading was compared against the MVC reading for each sensor. If the average EMG reading during any movement was higher than $6 \%$ of the MVC for that muscle, then the movement was thrown out of the results.

\subsubsection{Robot Dynamics and Gravity Compensation}

The $3 \times 3$ stiffness matrix found by multilinear regression does not represent passive wrist stiffness only. In fact, it is the combination of passive wrist stiffness, robot dynamics (stiffness, damping, etc.), and apparent stiffness due to gravity. The confounding effects were removed from the $3 \times 3$ stiffness tensor in the following manner.

Using the anthropometric data gathered from each subject, the subject's individual hand mass was calculated. An equivalent mass composed of calibrated weights was attached to the robot's hand interface, and the robot was commanded to duplicate the entire study using the deadweight affixed to the handle. The torque-position data gathered from this study was also reduced to a $3 \times 3$ stiffness matrix. This stiffness matrix originated from robot dynamics and apparent stiffness due to gravity alone. The subject's $3 \times 3$ stiffness tensor was compensated by subtracting the deadweight stiffness tensor, leaving a $3 \times 3$ stiffness matrix of the subject's passive wrist stiffness. 


\subsubsection{Matrix Decomposition}

While the $3 \times 3$ passive stiffness matrix is incredibly useful for dynamic simulations, it is not very approachable from a conceptual point of view; instead, other descriptive measures must be used that can be related to common observations. The basis of these descriptions is a result of the fact that the stiffness matrix may be represented as a three-dimensional ellipsoid, if it is symmetric. Unfortunately, the raw $3 \times 3$ stiffness matrix is asymmetric; however, it has been shown that the anti-symmetric portion is relatively small. This observation was checked for each of the subject's stiffness matrices by decomposing the matrix into symmetric and asymmetric components, and then by calculating the sum of forces that resulted from a uniform spherical distribution of unit displacements was made in the presence of the stiffness matrix. It was determined that the forces resulting from the anti-symmetric stiffness matrix were on average $0.03 \% \pm 0.01 \%$ as large as the forces that resulted from the symmetric stiffness matrix. The anti-symmetric portion was, therefore, discarded.

The symmetric portion of the stiffness matrix was used to plot ellipsoid visualizations of the stiffness tensor, as well as to calculate eigenvalues, eigenvectors, and angular orientations of the wrist stiffness. Further explanations of the descriptive measures calculated from the symmetric stiffness matrix and the statistical analysis performed on them can be found in the following chapter. 


\section{CENTRAL EXPERIMENT AND RESULTS}

Coordinated motor control requires that the neuromuscular system account and compensate for joint dynamics. Wrist movement dynamics are dominated by stiffness effects (Charles and Hogan, 2011). Wrist stiffness is anisotropic in nature, with greater stiffness in radial-ulnar deviation (RUD) than in flexion-extension (FE), and does not align with the anatomical axes of the wrist, coupling the wrist in FE and RUD (i.e. a movement in pure FE induces a torque in RUD, and vice versa) (Charles and Hogan, 2011). Because stiffness dominates wrist dynamics, these characteristics of passive wrist stiffness are significant and have an observable effect on wrist movement behavior (Charles and Hogan, 2010, 2012).

Most previous studies of wrist or forearm stiffness have measured passive joint stiffness in only 1 DOF, most often FE (Axelson and Hagbarth, 2001; De Serres and Milner, 1991; Gielen and Houk, 1984; Leger and Milner, 2000; Lehman and Calhoun, 1990). Few studies have measured stiffness in RUD (Rijnveld and Krebs, 2007), and only one study has measured stiffness in combinations of FE and RUD (Formica et al., 2012). Likewise, few studies have measure the passive stiffness in PS (Formica et al., 2012). Here we present the passive stiffness (i.e. in the absence of muscle activity) of the wrist and forearm for $15^{\circ}$ movements involving combinations of PS, FE, and RUD. 


\subsection{Methods}

The following section presents a summarized version of the methods used in conducting the principal study. Aspects of this section are discussed in greater detail in Chapter 3.

\subsubsection{Subjects}

Ten right-handed subjects (five female, $24 \pm 5.42$ years old (mean $\pm \mathrm{SD}$ ), range 18-38 years) with no history of neurological or biomechanical disorders affecting the wrist and forearm were recruited for the study. Because strenuous exercise may have a significant effect on passive joint stiffness (Leger and Milner, 2000; Ochi et al., 2007; Whitehead et al., 2003; Whitehead et al., 2001), subjects were instructed to refrain from strenuous upper body exercise for the 48 hour period preceding the study. Each subject gave informed consent consistent with the procedures outlined by BYU's Institutional Review Board.

\subsubsection{Experimental Setup}

An InMotion Wrist Rehabilitation Robot (Interactive Motion Technologies, MA) was used to measure stiffness. This robot is capable of moving the wrist and forearm independently in PS, FE, and RUD, senses displacement in each DOF at $200 \mathrm{~Hz}$ with a resolution of $0.0006^{\circ}$, and is able to apply $1.95 \mathrm{Nm}$ of torque in FE and RUD and $2.10 \mathrm{Nm}$ of torque in PS (Krebs et al., 2007). Subjects were seated at the robot with the right arm in the parasagittal plane, the shoulder in $0^{\circ}$ abduction, approximately $15^{\circ}$ flexion, and $0^{\circ}$ humeral rotation, and with the elbow in approximately $30^{\circ}$ of flexion. The robot manipulated the subjects' right wrist and forearm via a custom interface attached to the subjects' right hand. Because active gripping increases wrist stiffness through muscle activation (Hogan, 1985; Leger and Milner, 2000; Milner and Cloutier, 1993) and because finger constraint has been demonstrated to have a significant effect on the 
wrist's range-of-motion (ROM) (Gehrmann et al., 2008), the custom interface attached to the subject's hand along the palmar and dorsal aspects of the metacarpals, obviating the need for the subject to grip a handle and leaving the fingers unconstrained (See Figure 3-6). Each subject's distal forearm was attached with straps to the PS stage of the robot.

\subsubsection{Protocol}

We measured stiffness throughout the ROM extending $\pm 15^{\circ}$ from neutral position (defined below) in discrete movements combining PS, FE, and RUD. The robot rotated the forearm and wrist between neutral position and 134 targets equally distributed on the surface of a sphere of radius $15^{\circ}$ in PS-FE-RUD space (See Figure 4-1).

When muscle is stretched more than $1 \%$ of its length from rest, the stiffness during the initial portion of the movement is significantly higher than during the rest of the movement (Campbell, 2010). For the wrist, this short-range-stiffness (SRS) effect has been observed over the initial $3-4^{\circ}$ of wrist rotation (Axelson and Hagbarth, 2001). To allow us to later remove the SRS, additional $3^{\circ}$ bands were added to the beginning and end of each movement. The resulting outbound movement was programmed to begin at $-3^{\circ}$ relative to the neutral position, pass through the neutral position, and end at $+18^{\circ}$ relative to neutral position. The inbound movement extended from $+18^{\circ}$ through the origin to $-3^{\circ}$. Because the robot was commanded via proportional-derivative control (with proportional and derivative gains of $10.0 \mathrm{Nm} / \mathrm{rad}$ and 0.1 $\mathrm{Nms} / \mathrm{rad}$ for FE and RUD, and gains of $20.0 \mathrm{Nm} / \mathrm{rad}$ and $0.3 \mathrm{Nms} / \mathrm{rad}$ for PS), the actual displacement was generally less than the commanded displacement by approximately $20 \%$. Each movement was repeated three times in succession before proceeding to the next target. The session lasted a total of 50 minutes. 

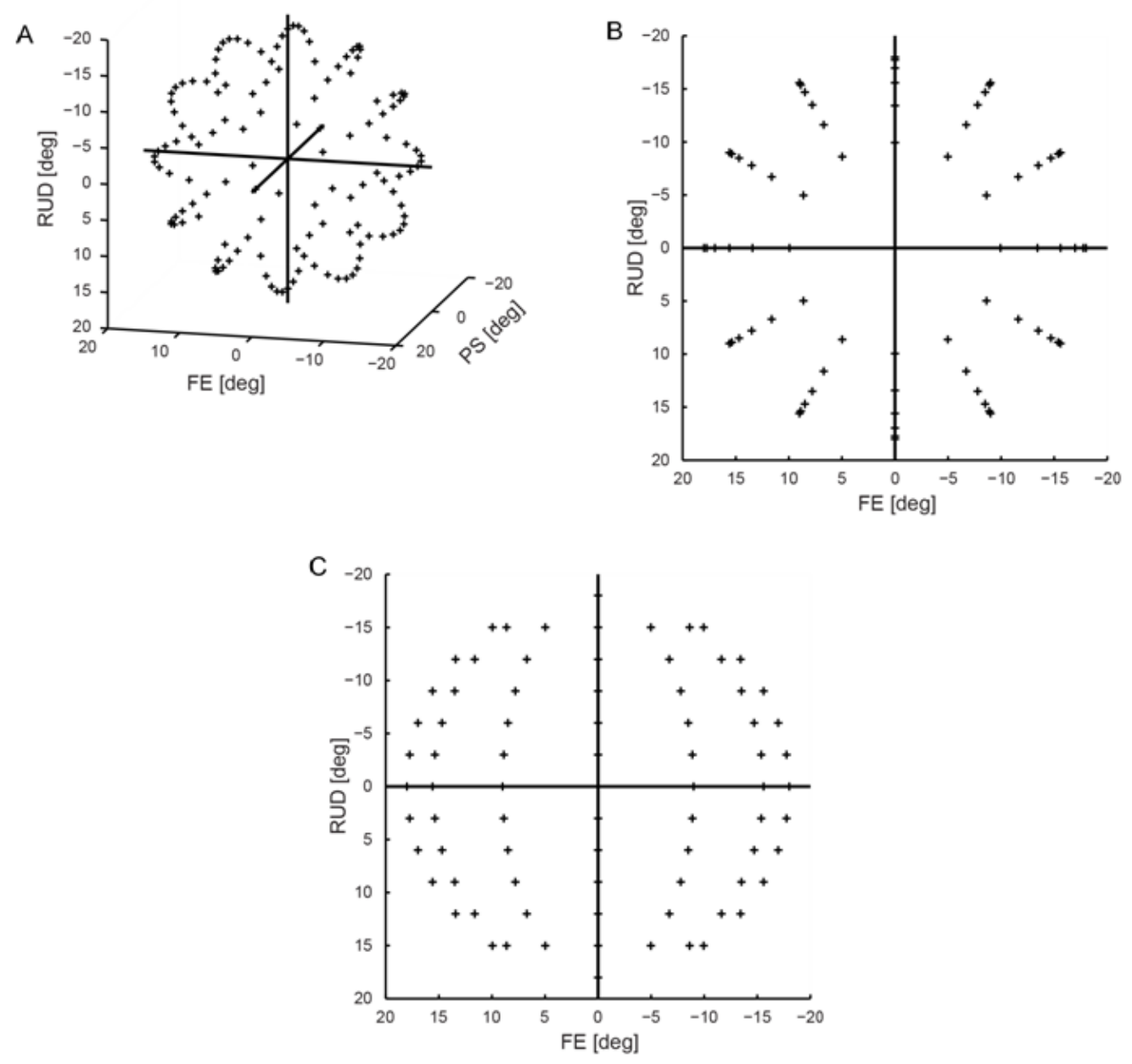

Figure 4-1: Plots of the point distribution used during the study. The robot was programmed to move from neutral position (origin) to each of these 134 positions in PS-FE-RUD space. A) Isometric view. B) The 12 divisions in FE-RUD space. C) The 13 divisions along the PS axis. P, F, and UD represent pronation, flexion, and ulnar deviation, respectively (negative values of $P, F$, and UD represent supination, extension, and radial deviation, respectively).

To avoid stimulating reflexes, the robot was programmed to move slowly and smoothly (following a minimum jerk trajectory with average velocity of $5.2 \mathrm{deg} / \mathrm{sec}$ ). Subjects were instructed to relax their upper arm as much as possible during the duration of the session. We verified that muscles were relaxed by recording surface EMG at $200 \mathrm{~Hz}$ (Trigno by Delsys, Boston, MA) in the flexor carpi radialis, flexor carpi ulnaris, extensor carpi radialis, extensor carpi ulnaris, pronator teres, and biceps during each session (the biceps is the supination muscle 
most accessible by surface EMG). Following completion of the session, three sets of maximum voluntary contraction (MVC) EMG measurements were obtained for each muscle.

Neutral position was defined similar to ISB recommendations (Wu et al., 2005) for global movements, with approximations for in vivo use. The forearm was in neutral PS when the dorsal aspect of the distal forearm (the dorsal tubercle of the radius and the dorsal-most protuberance of the ulnar head) was parallel to the vertical plane containing the arm and forearm. FE and RUD were defined to be in neutral position when the long axis of the third metacarpal aligned with the long axis of the forearm. The wrist was in neutral FE and RUD when the elbow joint center, the wrist joint center, and the center of the head of the third metacarpal were aligned. The subject was placed in the neutral position by changing the zero position of the robot.

\subsubsection{Data Processing}

The wrist robot output included joint torques (calculated from motor voltages) and displacements for FE, RUD, and PS. After removing the SRS effects (observed primarily in the first $2^{\circ}$ of movement), the data were separated into outbound and inbound movements (to avoid the effects of hysteresis) and shifted to the origin.

The torque-displacement data formed a vector field of torques, $\vec{\tau}\left(\theta_{P S}, \theta_{F E}, \theta_{R U D}\right)$, at different values of PS, FE, and RUD $\left(\theta_{P S}, \theta_{F E}\right.$, and $\left.\theta_{R U D}\right)$. The 3-DOF stiffness matrix $K$ was derived by linearizing the torque vector $\vec{\tau}$ by Taylor series expansion: 


$$
\begin{gathered}
{\left[\begin{array}{c}
\tau_{P S} \\
\tau_{F E} \\
\tau_{R U D}
\end{array}\right]=\left[\begin{array}{c}
\frac{\partial \tau_{P S}}{\partial \theta_{P S}} \theta_{P S}+\frac{\partial \tau_{P S}}{\partial \theta_{F E}} \theta_{F E}+\frac{\partial \tau_{P S}}{\partial \theta_{R U D}} \theta_{R U D} \\
\frac{\partial \tau_{F E}}{\partial \theta_{P S}} \theta_{P S}+\frac{\partial \tau_{F E}}{\partial \theta_{F E}} \theta_{F E}+\frac{\partial \tau_{F E}}{\partial \theta_{R U D}} \theta_{R U D} \\
\frac{\partial \tau_{R U D}}{\partial \theta_{P S}} \theta_{P S}+\frac{\partial \tau_{R U D}}{\partial \theta_{F E}} \theta_{F E}+\frac{\partial \tau_{R U D}}{\partial \theta_{R U D}} \theta_{R U D}
\end{array}\right]=\left[\begin{array}{ccc}
\frac{\partial \tau_{P S}}{\partial \theta_{P S}} & \frac{\partial \tau_{P S}}{\partial \theta_{F E}} & \frac{\partial \tau_{P S}}{\partial \theta_{R U D}} \\
\frac{\partial \tau_{F E}}{\partial \theta_{P S}} & \frac{\partial \tau_{F E}}{\partial \theta_{F E}} & \frac{\partial \tau_{F E}}{\partial \theta_{R U D}} \\
\frac{\partial \tau_{R U D}}{\partial \theta_{P S}} & \frac{\partial \tau_{R U D}}{\partial \theta_{F E}} & \frac{\partial \tau_{R U D}}{\partial \theta_{R U D}}
\end{array}\right]\left[\begin{array}{c}
\theta_{P S} \\
\theta_{F E} \\
\theta_{R U D}
\end{array}\right]} \\
=\left[\begin{array}{ccc}
K_{P S, P S} & K_{P S, F E} & K_{P S, R U D} \\
K_{F E, P S} & K_{F E, F E} & K_{F E, R U D} \\
K_{R U D, P S} & K_{R U D, F E} & K_{R U D, R U D}
\end{array}\right]\left[\begin{array}{c}
\theta_{P S} \\
\theta_{F E} \\
\theta_{R U D}
\end{array}\right]=K\left[\begin{array}{c}
\theta_{P S} \\
\theta_{F E} \\
\theta_{R U D}
\end{array}\right]
\end{gathered}
$$

The subject-specific values for each row of the total measured stiffness matrix $K$ were computed by multiple linear regression (using Matlab's regress function):

$$
\begin{array}{rll}
{\left[\begin{array}{lllll}
K_{P S, P S} & K_{P S, F E} & K_{P S, R U D}
\end{array}\right]=\operatorname{regress}\left(\tau_{P S},\left[\begin{array}{llll}
1 & \theta_{P S} & \theta_{F E} & \theta_{R U D}
\end{array}\right]\right)} \\
{\left[\begin{array}{lllll}
K_{F E, P S} & K_{F E, F E} & K_{F E, R U D}
\end{array}\right]=\operatorname{regress}\left(\tau_{F E},\left[\begin{array}{llll}
1 & \theta_{P S} & \theta_{F E} & \theta_{R U D}
\end{array}\right]\right)} \\
{\left[\begin{array}{lllll}
K_{R U D, P S} & K_{R U D, F E} & K_{R U D, R U D}
\end{array}\right]=\operatorname{regress}\left(\tau_{R U D},\left[\begin{array}{llll}
1 & \theta_{P S} & \theta_{F E} & \theta_{R U D}
\end{array}\right]\right)}
\end{array}
$$

The measured robot torques do not originate solely from passive joint stiffness, but represent the sum of torques from 1) passive wrist stiffness, 2) mechanical impedance in the robot, and 3) apparent stiffness due to gravity. Consequently, the stiffness matrix would include apparent stiffness from all three phenomena and not the passive wrist stiffness only. Therefore, we removed the two latter effects in the following manner. A stiffness compensation matrix representing only robot-based and gravity-induced effects was measured for each subject by repeating the experimental protocol with a mass attached to the robot instead of the subject's hand. The mass was matched to that of each subject's hand using anthropometric measurements and regression equations from de Leva (de Leva, 1996), and the position of the mass in the robot 
and the neutral position of the robot were matched to each subject's condition. We then subtracted the subject-specific stiffness compensation matrix from each subject's total measured stiffness matrix to yield the passive wrist stiffness matrix.

The measured stiffness matrix contains both conservative and non-conservative components, but pure spring-like behavior is perfectly conservative (Hogan, 1985; Mussa-Ivaldi et al., 1985). We therefore separated the measured stiffness into its conservative (symmetric) and non-conservative (anti-symmetric) parts. The anti-symmetric portion of the stiffness matrix is generally negligible compared to the symmetric portion and may therefore be disregarded. We compared the relative sizes of the symmetric and anti-symmetric portions by comparing the sum of the magnitudes of the torques arising from either portion for 134 unit displacements equally distributed throughout PS-FE-RUD space.

All EMG data were detrended, rectified, and low-pass filtered using a $2^{\text {nd }}$ order Butterworth filter with a cutoff frequency of $3 \mathrm{~Hz}$. The maximum values of processed EMG from each of the three processed MVC data sets were averaged to establish the MVC value for each of the six muscles for each subject. A subject was considered to not be relaxed if the average EMG reading during a movement exceeded $6 \%$ of the individual's MVC. Precise methods for EMG analysis have not been established either formally or informally for wrist motion studies. Some researchers averaged EMG over the entire length of the study (Formica et al., 2012), resulting in a low average EMG but excluding the possibility to identify and remove small sections of subject interference. Others give no numerical analysis of EMG or numerical proof of subject's passivity (Rijnveld and Krebs, 2007). Of the 2040 movements recorded throughout the study, $99 \%$ fell below the $6 \%$ MVC threshold. The remaining movements were excluded from the analysis. 


\subsubsection{Analysis}

A symmetric stiffness matrix is more intuitively analyzed in terms of the shape, orientation, and volume of its stiffness ellipsoid. The directions and magnitudes of the principal axes of the stiffness ellipsoid are given by the eigenvectors and eigenvalues of the stiffness matrix, respectively. We characterized the shape of the ellipsoid by the ratios of the intermediate

and minor eigenvalues with respect to the major eigenvalue: $\lambda_{2} / \lambda_{1}$ and $\lambda_{3} \lambda_{1}$, where $\lambda_{1}, \lambda_{2}$, and $\lambda_{3}$ are the major, intermediate, and minor eigenvalues, respectively.. The orientation of the ellipsoid was determined as the angles between the principal axes and the PS, FE, and RUD axes. The volume of the ellipsoid represents the overall magnitude of the passive stiffness and was calculated as $\frac{4}{3} \pi \lambda_{1} \lambda_{2} \lambda_{3}$.

To determine the effect of experimental conditions on ellipsoid shape, we performed a three-way ANOVA for the ratios of intermediate to major eigenvalue and minor to major eigenvalue, with movement repetition (first, second, or third pass), movement direction (outbound or inbound), and gender as fixed factors. The ANOVA was then repeated using movement repetition and movement direction as fixed factors and subject as a random factor. Similar pairs of three-way ANOVAs were performed for ellipsoid volume and the angle between the major eigenvector and the RUD axis. 


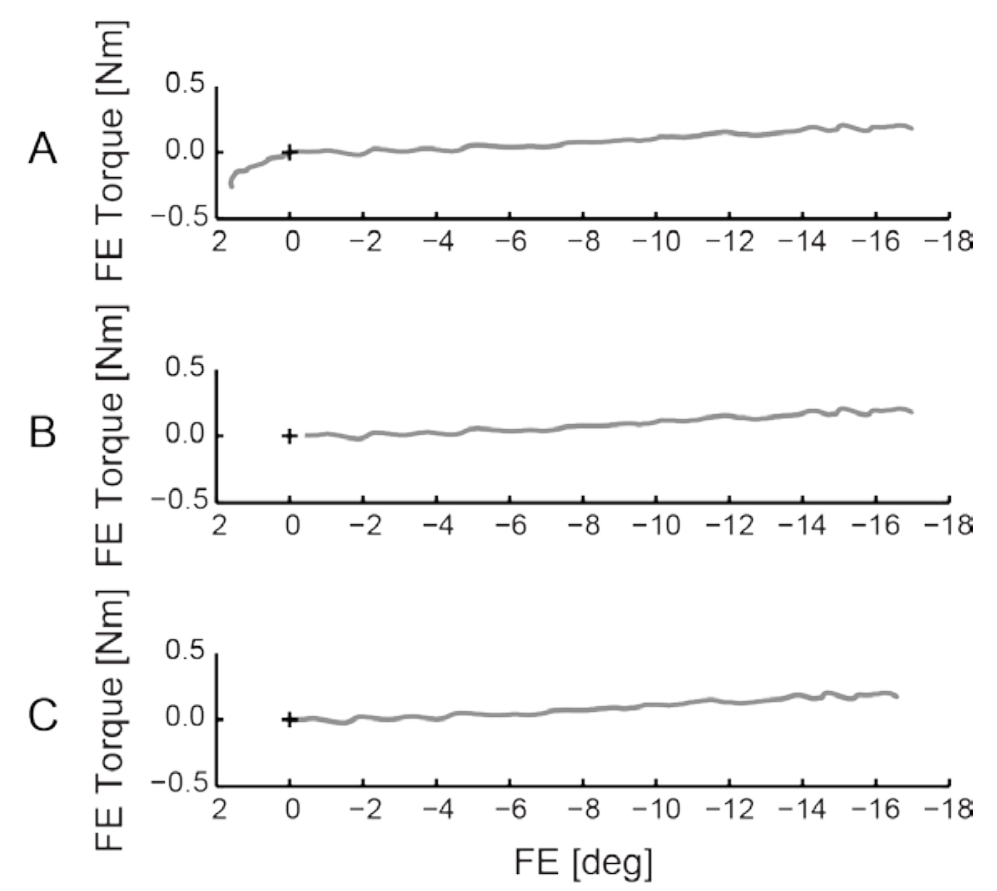

Figure 4-2: Progression of the torque-displacement data from raw data to processed data. A) Raw torque-displacement data from a typical movement in pure extension. B) The initial $2^{\circ}$ of displacement were removed to eliminate short-range stiffness effects. C) The data were then shifted to the origin to allow simultaneous linear regression across all movement directions. $F$ represents flexion, and $\mathrm{F}$ Torque represents the torque in flexion, with negative values representing extension and the torque in extension, respectively.

\subsection{Results}

Consistent with previous findings (Axelson and Hagbarth, 2001), we observed the short-rangestiffness phenomenon in approximately the first $2^{\circ}$ of displacement (Figure 4-2) and excluded it from the analysis, leaving an average displacement of $14.69 \pm 1.04^{\circ}$ in each direction.

The multiple linear regression showed a linear relationship between torque and displacement: the mean r-squared value for each row of the stiffness matrix (before accounting for robot and gravitational effects) was found to be $0.90 \pm 0.04$ (range 0.82-0.98). After compensating for gravity and robot dynamics, the matrices were separated into symmetric and anti-symmetric parts. On average, the sum of the magnitude of the resultant torques due to the anti-symmetric matrix was $0.03 \% \pm 0.01 \%$ of that produced by the symmetric matrix. The anti- 
symmetric matrix was excluded from further analysis, yielding the symmetric stiffness matrices shown in Table 4-1.

Table 4-1: Mean compensated stiffness matrices and standard deviations for subsets of the study. The $R^{2}$ values shown give the correlation of the matrices to the original data prior to compensation.

\begin{tabular}{|c|c|c|c|c|c|c|c|c|c|}
\hline & & & \multirow{2}{*}{\multicolumn{3}{|c|}{ Mean $[\mathrm{Nm} / \mathrm{rad}]$}} & \multirow{2}{*}{\multicolumn{3}{|c|}{ St. Dev. [Nm/rad] }} & \multirow{3}{*}{$\mathrm{R}^{2}$} \\
\hline & & & & & & & & & \\
\hline & & & $\mathrm{P}$ & $\mathrm{F}$ & $\mathrm{U}$ & $\mathrm{P}$ & $\mathrm{F}$ & $\mathrm{U}$ & \\
\hline \multirow{6}{*}{$\sum_{\Sigma}^{\frac{0}{\pi}}$} & \multirow{3}{*}{ Outbound } & $\mathrm{P}$ & 0.75 & 0.02 & 0.29 & 0.24 & 0.15 & 0.19 & $0.85-0.88$ \\
\hline & & $\mathrm{F}$ & 0.02 & 1.00 & -0.12 & 0.15 & 0.23 & 0.13 & $0.92-0.94$ \\
\hline & & $\mathrm{U}$ & 0.29 & -0.12 & 3.00 & 0.19 & 0.13 & 0.90 & $0.92-0.97$ \\
\hline & \multirow{3}{*}{ Inbound } & $\mathrm{P}$ & 0.70 & 0.03 & 0.28 & 0.21 & 0.12 & 0.16 & $0.86-0.88$ \\
\hline & & $\mathrm{F}$ & 0.03 & 0.92 & -0.06 & 0.12 & 0.25 & 0.09 & $0.86-0.91$ \\
\hline & & $\mathrm{U}$ & 0.28 & -0.06 & 2.55 & 0.16 & 0.09 & 0.79 & $0.95-0.98$ \\
\hline \multirow{6}{*}{$\frac{\tilde{J}}{\tilde{J}}$} & \multirow{3}{*}{ Outbound } & $\mathrm{P}$ & 0.87 & 0.07 & 0.15 & 0.74 & 0.11 & 0.05 & $0.82-0.91$ \\
\hline & & $\mathrm{F}$ & 0.07 & 0.76 & -0.09 & 0.11 & 0.16 & 0.10 & $0.91-0.92$ \\
\hline & & $\mathrm{U}$ & 0.15 & -0.09 & 2.36 & 0.05 & 0.10 & 0.39 & $0.91-0.97$ \\
\hline & \multirow{3}{*}{ Inbound } & $\mathrm{P}$ & 0.63 & 0.10 & 0.13 & 0.46 & 0.12 & 0.04 & $0.83-0.89$ \\
\hline & & $\mathrm{F}$ & 0.10 & 0.70 & 0.01 & 0.12 & 0.09 & 0.13 & $0.85-0.90$ \\
\hline & & $\mathrm{U}$ & 0.13 & 0.01 & 1.96 & 0.04 & 0.13 & 0.21 & $0.89-0.94$ \\
\hline
\end{tabular}

The shape of the stiffness ellipsoids was highly anisotropic (Figure 4-3; Table 4-2): on average, the ratios of intermediate to major eigenvalue and minor to major eigenvalue were $0.43 \pm 0.11$ and $0.21 \pm 0.09$, respectively. In contrast, the intermediate and minor axes were similar in magnitude (the average the ratio of minor to intermediate eigenvalue was $0.49 \pm 0.17$ ). The ratio of intermediate to major eigenvalue was significantly dependent on gender $(p=0.002)$ but did not depend on repetition $(p=0.83)$ or direction of movement $(p=0.81)$. Conversely, the ratio of minor to major eigenvalue was not significantly dependent on gender $(\mathrm{p}=0.22)$, but was statistically dependent (although only slightly) on direction of movement $(p=0.025)$, again with no effect of repetition $(p=0.42)$. 
A

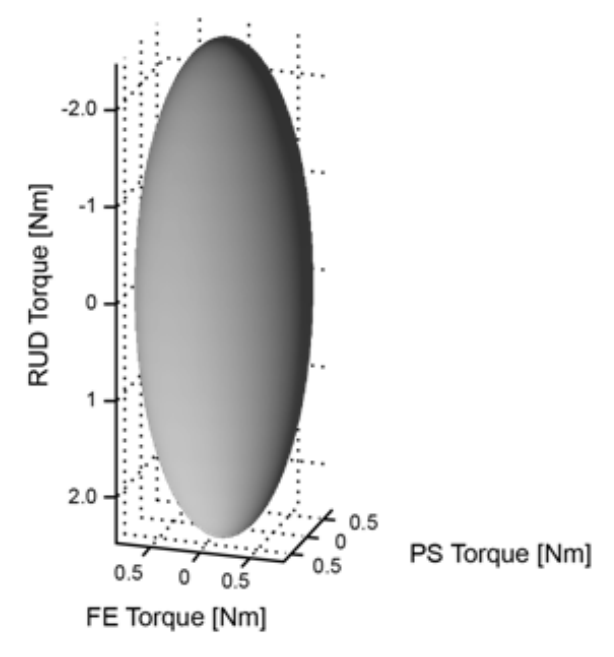

B

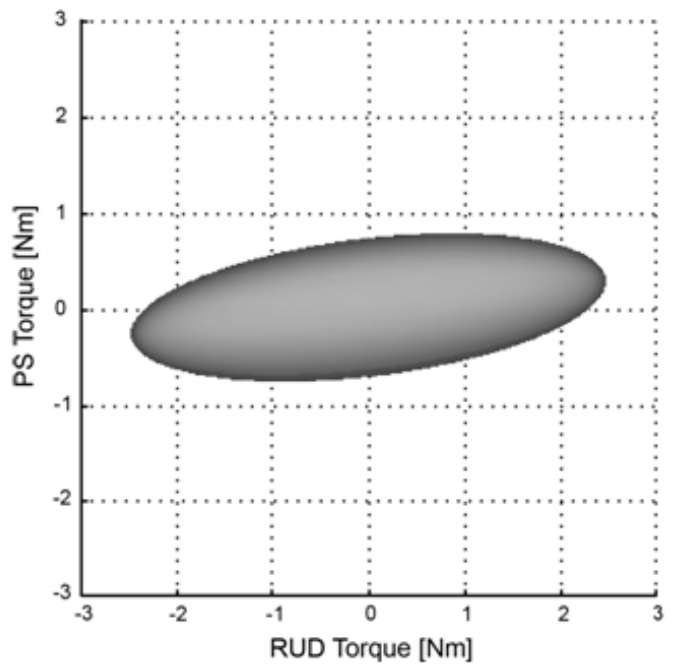

C

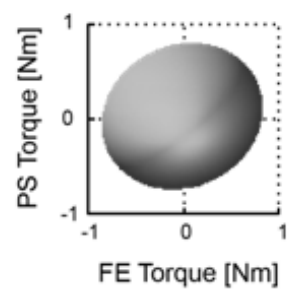

Figure 4-3: Stiffness matrices displayed as stiffness ellipsoids. The ellipsoid shown is the stiffness for outbound movements averaged over all subjects. A) Isometric view. B) Stiffness is significantly greater in RUD than in PS (and FE, not shown). C) Stiffness is similar in FE and PS. P Torque, F Torque, and UD Torque represent the torque in pronation, flexion, and ulnar deviation, respectively (negative values represent the torque in supination, extension, and radial deviation, respectively).

Table 4-2: Comparison of ellipsoid shape, orientation, and volume by gender and direction (inbound vs. outbound). Shape is given as the ratio of eigenvalues (maj, int, and min represent major, intermediate, and minor eigenvalues, respectively). Orientation is given as the angle between the major eigenvector and the RUD axis.

\begin{tabular}{|c|c|c|c|c|c|c|c|c|c|}
\hline & \multicolumn{2}{|c|}{ Min/Maj } & \multicolumn{2}{|c|}{ Int/Maj } & \multicolumn{2}{|c|}{ Angle [deg] } & \multicolumn{2}{|c|}{$\begin{array}{c}\text { Volume } \\
{\left[(\mathrm{Nm} / \mathrm{rad})^{3}\right]}\end{array}$} \\
\hline & & Mean & St. Dev. & Mean & St. Dev. & Mean & St. Dev. & Mean & St. Dev. \\
\hline \multirow{2}{*}{$\frac{0}{\sum^{\pi}}$} & Outbound & 0.22 & 0.08 & 0.37 & 0.07 & 8.40 & 2.83 & 9.11 & 4.89 \\
\hline & Inbound & 0.24 & 0.09 & 0.39 & 0.06 & 9.45 & 3.32 & 6.65 & 3.80 \\
\hline \multirow{2}{*}{ 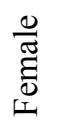 } & Outbound & 0.19 & 0.11 & 0.48 & 0.17 & 8.54 & 5.10 & 6.40 & 6.20 \\
\hline & Inbound & 0.20 & 0.10 & 0.46 & 0.08 & 7.71 & 3.44 & 3.33 & 2.64 \\
\hline
\end{tabular}

The orientation of the major eigenvector pointed roughly in the direction of pure RUD for all subjects. The angle between the average major eigenvector and the RUD axis was $8.53^{\circ}$. The average intermediate eigenvector fell within $13.7^{\circ}$ of the FE axis. The minor eigenvector was not 
aligned with the anatomical axes (while eigenvectors are mutually orthogonal, the means of eigenvectors are not) (Figure 4-4; Table 4-2). The direction of the major eigenvector forced the intermediate and minor eigenvectors to lie roughly in the FE-PS plane, with no significant correlation between the minor or the intermediate eigenvectors and the PS or FE axes, demonstrated by the finding that there was no significant difference $(p=0.45)$ between the angle formed by the minor eigenvector and PS axis and $45^{\circ}$ (the angle expected for a random distribution).

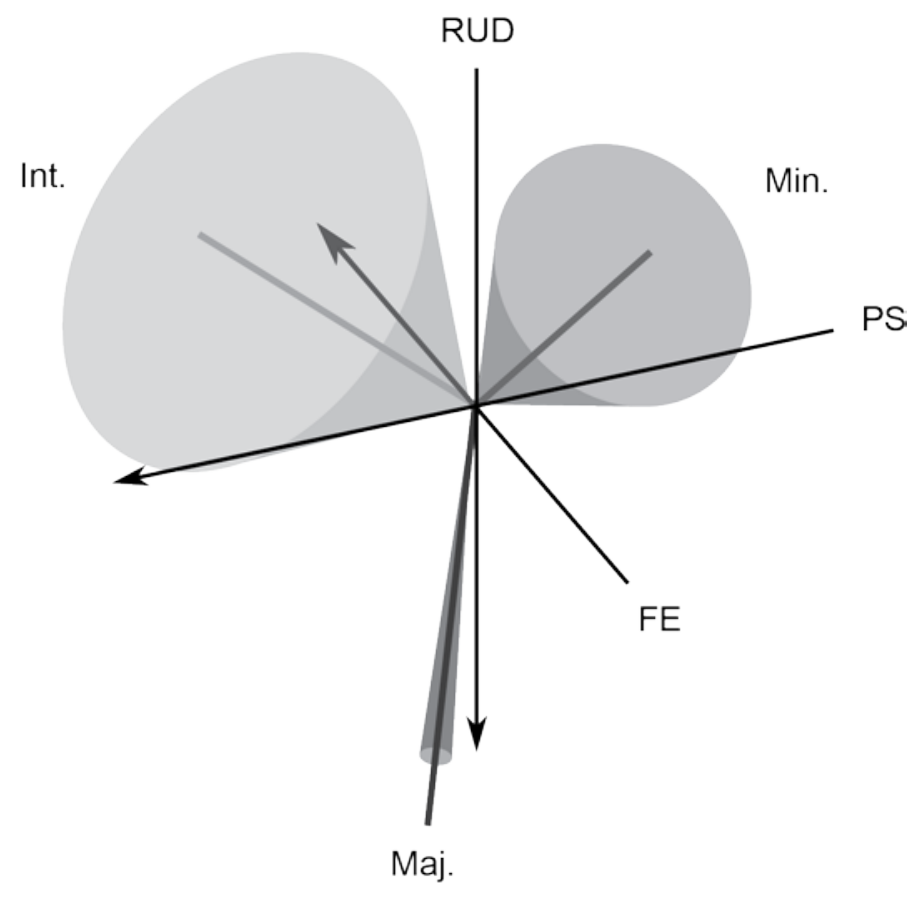

Figure 4-4: Mean and standard deviation of eigenvector orientation for all stiffness ellipsoids (inbound and outbound). Heavy black lines show the mean orientation for the major, intermediate, and minor eigenvectors (Maj, Int, and Min, respectively). The angle of each cone is the standard deviation of the angles between the eigenvectors of the individual and mean stiffness matrices and represents the spread of eigenvector directions. $P, F$, and UD represent pronation, flexion, and ulnar deviation, respectively.

The ellipsoid volume was significantly larger for outbound than for inbound movements $(\mathrm{p}<0.001)$ and for male than for female subjects $(\mathrm{p}=0.015$; Figure 4-5; Table 4-2). The average volume for inbound movements was $64 \%$ of the volume for outbound movements, and the 
average volume for female subjects was $62 \%$ of the volume for male subjects (note that reporting differences in stiffness magnitude by comparing differences in volume emphasizes the differences because volume is proportional to the product of the eigenvalues; for example, while the average female volume is only $64 \%$ of the average male volume, the eigenvalues of the average female ellipsoid are $95 \%, 85 \%$, and $77 \%$ as large as those of the average male ellipsoid). There was no significant effect of repetition on volume $(p=0.94)$.

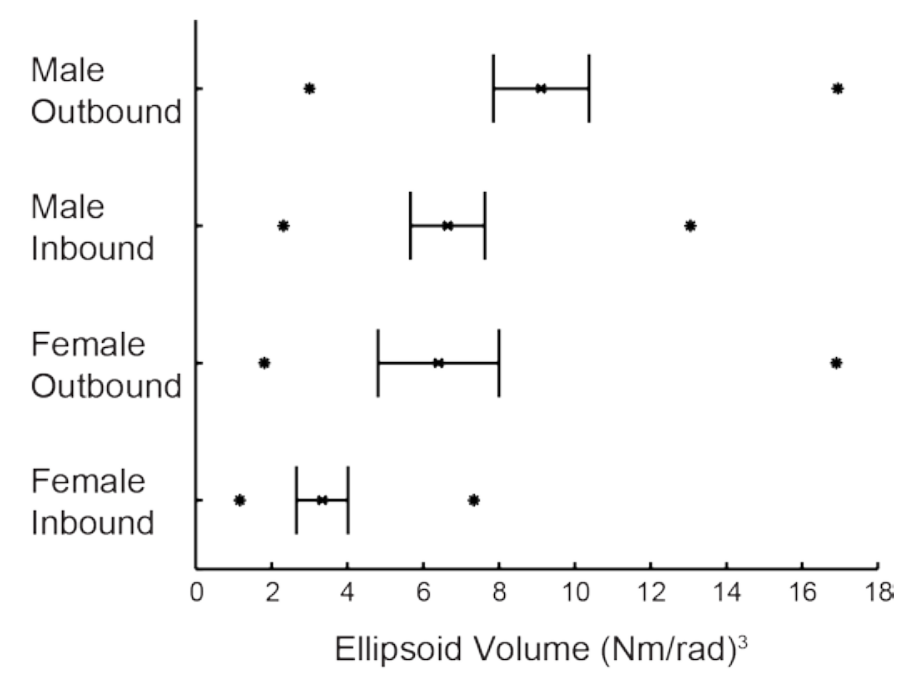

Figure 4-5: Mean, standard error, and range (minimum and maximum values) of the volume of stiffness ellipsoids by gender and direction (outbound vs. inbound).

\subsection{Discussion}

Coordinated motor control requires that the neuromuscular system account and compensate for joint dynamics. Recent studies have shown that stiffness dominates wrist dynamics (Charles and Hogan, 2011, 2012); more specifically, wrist stiffness is anisotropic (Formica et al., 2012), creating "paths of least resistance" which may be exploited by the neuromuscular system when it has the opportunity—-for example, when the three degrees of freedom of the wrist and forearm are used to complete a task that only requires two degrees of freedom. Understanding how wrist and forearm rotations are coordinated requires an understanding of the stiffness encountered 
during combined wrist and forearm rotations. Here we have characterized the passive stiffness of coupled wrist and forearm rotations of $15^{\circ}$ amplitude and found that the stiffness is highly anisotropic (the major eigenvector is approximately 5 times larger than the minor eigenvector), the major eigenvector being closely aligned with the RUD axis.

\subsubsection{Comparison to Prior Measurements}

Almost all prior investigations of wrist or forearm stiffness have reported stiffness values for movement in a single degree of freedom only, most often FE. The diagonal values of the stiffness matrices reported here represent the passive stiffness along the anatomical axes (PS, FE, and RUD) and were compared to those of previous studies. The mean stiffness in FE measured in this study $(0.85 \mathrm{Nm} / \mathrm{rad})$ falls in the middle of those reported in previous studies, which range from $0.15 \mathrm{Nm} / \mathrm{rad}$ (Lehman and Calhoun, 1990) to $3 \mathrm{Nm} / \mathrm{rad}$ (De Serres and Milner, 1991). Our mean stiffness measurement in RUD ( $2.47 \mathrm{Nm} / \mathrm{rad})$ is comparable to the two previous measurements of passive stiffness in RUD: $1.45 \mathrm{Nm} / \mathrm{rad}$ (Rijnveld and Krebs, 2007) and 1.74 $\mathrm{Nm} / \mathrm{rad}$ (Formica et al., 2012). Finally, our mean value of stiffness in PS $(0.74 \mathrm{Nm} / \mathrm{rad})$ is of the same order of magnitude as the only previously published values of $0.19 \mathrm{Nm} / \mathrm{rad}$ in pronation and $0.24 \mathrm{Nm} / \mathrm{rad}$ in supination (Formica et al., 2012).

Only one previously reported study investigated passive wrist stiffness in directions involving both FE and RUD (Formica et al., 2012). We compared the stiffness ellipse from that study with the stiffness ellipse obtained from the FE-RUD portion of our 3-DOF stiffness matrix and found that our FE-RUD ellipse is more anisotropic (ratio of minor to major eigenvector of 0.34 vs. 0.68 in the previous study) and more aligned with the FE-RUD axes (angle between major eigenvector and RUD axis of $2.2 \pm 4.1^{\circ}$ vs. $21 \pm 9.2^{\circ}$ ), as shown in Figure 4-6 A-B. 
The differences in measured stiffness may have been caused by the following differences between the present and previous experiments. First, we defined the origin to be in neutral wrist and forearm position (see Methods). In contrast, in the previous study the origin was defined with the upper limb in a typical posture for grasping a handle (Figure 4-6 C). The shoulder was abducted by approximately $40^{\circ}$, effectively supinating the forearm by $40^{\circ}$. Rotating the forearm was recently shown to counter-rotate the pulling directions of the wrist muscles by $12 \%$ relative to a frame intrinsic to the wrist (Ojima et al., 1992), suggesting that the orientation of the ellipse in the previous study was pronated by approximately $5^{\circ}$ relative to the present study. Also, in the previous study the origin in PS was determined by the orientation of the forearm when the hand was grasping the vertically oriented robot handle. Compared to neutral PS position (see Methods), grasping the handle pronated the forearm by $6.1 \pm 3.2^{\circ}$ (measured on six of the subjects from the present study). In FE and RUD, the origin was defined by the handle, which was in line with the forearm in the parasagittal plane (forcing the wrist into an extended position) and inclined relative to the horizontal plane by $17^{\circ}$. Compared to our study, this origin was in $19.9 \pm 4.2^{\circ}$ of extension and $1.5 \pm 4.6^{\circ}$ of ulnar deviation (measured on six of the subjects from the present study).

Second, robot dynamics and gravitational effects added a substantial amount of apparent stiffness, meriting great care in estimating and removing their contribution. Here we went beyond the efforts of the previous study by estimating these effects separately for each subject (based on each subject's hand mass and neutral position). Third, we compared the 2-DOF stiffness from the previous study to a subset of the 3-DOF matrix from the present study. Because this subset came from a linear regression across the entire 3-DOF space, it is influenced by the third DOF and is therefore not as specific to the 2-DOF space as the previous 
measurement. Fourth, in the previous study, the fingers were constrained in a flexed position (around a handle), presumably increasing the measurement stiffness in FE relative to our study.

A

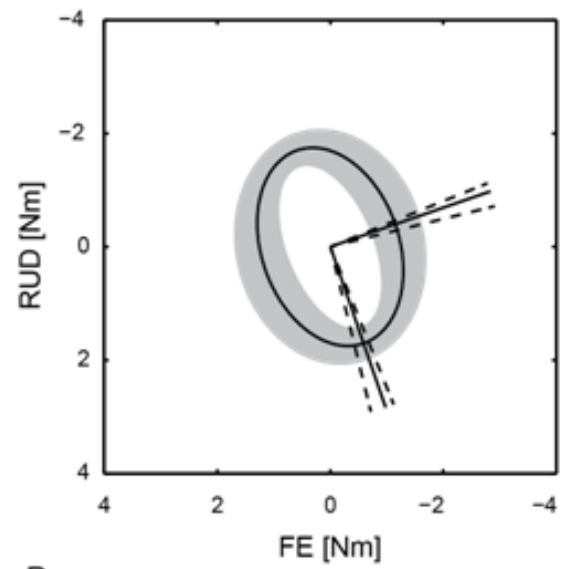

B

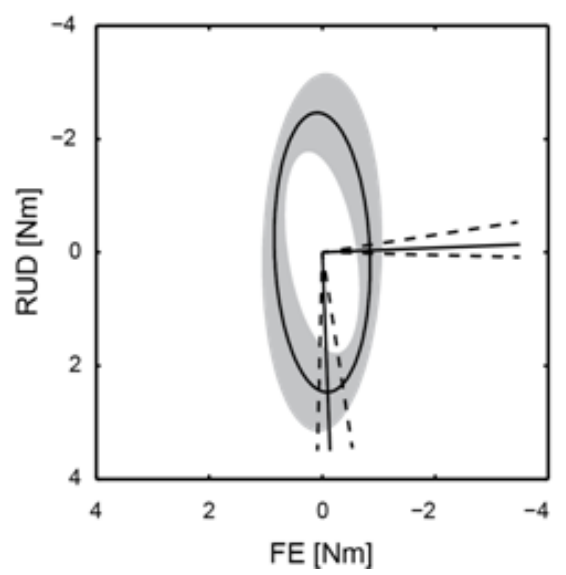

Figure 4-6: Comparison of our (3-DOF) results to prior measurements of wrist stiffness (in 2 DOF). A-B) Mean stiffness ellipse in FE-RUD for A) the current study and B) Formica et al. (Formica et al., 2012). The line represents the mean ellipse; the inside and outside border of the shaded region represent the ellipses of the mean \pm standard deviation matrices. C) Differences in the origin of the stiffness measurements between the current study and (Formica et al., 2012). The measurement space in the current (3-DOF) study is represented by the sphere and is centered in neutral anatomical position. The measurement space in the (2-DOF) study by (Formica et al., 2012) is represented by the circle (contained in an FE-RUD plane) and is centered at approximately -34', $20^{\circ}$, and $1.5^{\circ}$ in $\mathrm{P}, \mathrm{F}$, and $\mathrm{UD}$, respectively. 


\subsubsection{Implications for the Neural Control of Movement}

The anisotropy in stiffness creates a control challenge because it causes path curvature unless the muscular activation patterns specifically compensate for the anisotropy (Charles and Hogan, 2010, 2012). The anisotropy between RUD and FE measured here is approximately twice as great as previously reported (Formica et al., 2012) and would result in more path curvature (and a greater control challenge for making straight paths) than previously thought.

The coupling between FE and RUD reported here $\left(2^{\circ}\right.$, measured as the angle between the principal stiffness axes and the anatomical axes) is smaller than previously reported $\left(21^{\circ}\right)$ in (Formica et al., 2012). Coupling between FE and RUD means that a movement in pure FE will require a torque in RUD (in addition to the torque in FE), and vice versa. From the point of view of neural control, the amount of coupling between FE and RUD is not critically important because almost all wrist movements involve both FE and RUD and therefore require torques in both DOF anyway. The large variability in the magnitude of stiffness (ellipsoid volume) between subjects is similar to the large variability in body segment inertia and has been discussed previously (Formica et al., 2012).

As mentioned in the introduction, the anisotropy in wrist stiffness creates paths of least resistance which could be exploited by using PS to orient the wrist to use FE instead of RUD (assuming the stiffness in PS is small enough to make this substitution worthwhile). Here we have shown that the stiffness in PS is significantly smaller than the stiffness in RUD, indicating that the torque required to move in combinations of PS and FE is significantly smaller than the torque required to move in combinations of FE and RUD (assuming equal displacements). The coupled stiffness measured here will enable future research to determine optimal paths and to compare these optimal paths to observed movements involving wrist and forearm rotations. 


\section{DISCUSSION}

An accurate and complete characterization of wrist joint dynamics is essential for understanding and addressing the many health-related issues surrounding the wrist joint. Because passive wrist stiffness has the greatest effect on the passive dynamics of the wrist, any complete characterization must include a thorough study of the factors contributing to passive stiffness. Furthermore, because natural wrist movement occurs in coordination with forearm movement, understanding wrist movements relies on an understanding of forearm stiffness as well. Whereas previous research investigating the passive stiffness of the wrist and forearm has been limited to one or two degrees-of-freedom (DOF), the current research has endeavored to characterize passive stiffness in all three DOF of the wrist and forearm.

Our principle experiment measured passive stiffness throughout the range $\pm 15^{\circ}$ from the neutral position in FE, RUD, and PS. This was accomplished with the aid of a wrist and forearm rehabilitation robot (Interactive Motion Technologies, MA) that was programmed to move the subject's wrist and forearm through three simultaneous repetitions of movement-origin to target displacement and back - through 134 combinations of simultaneous FE, RUD, and PS movement. Ten subjects with no history of neurological or biomechanical disorder affecting the wrist or forearm were tested $(24 \pm 5.4$ years old, range $18-38)$. The torque data gathered during these experiments was reduced to a symmetric $3 \times 3$ stiffness matrix, which gives a linear estimation of the stiffness throughout the tested range-of-motion (ROM). 
Table 5-1: Comparison of our (3-DOF) results to prior measurements of wrist stiffness (in 2 DOF).

\begin{tabular}{|l|c|c|c|c|c|c|}
\cline { 2 - 7 } \multicolumn{1}{l|}{} & FE/FE & RUD/RUD & PS/PS & RUD/FE & PS/FE & PS/RUD \\
\hline (Rijnveld and Krebs, 2007) & -- & 1.45 & -- & -- & -- & -- \\
\hline $\begin{array}{l}\text { (Lehman and Calhoun, } \\
\text { 1990) }\end{array}$ & $0.002-0.15$ & -- & -- & -- & -- & -- \\
\hline $\begin{array}{l}\text { (Axelson and Hagbarth, } \\
\text { 2001) }\end{array}$ & 0.34 & -- & -- & -- & -- & -- \\
\hline (Gielen and Houk, 1984) & $0.32-0.70$ & -- & -- & -- & -- & -- \\
\hline (Pando et al.) & 0.84 & 2.02 & -- & -0.27 & -- & -- \\
\hline (Drake and Charles) & $\mathbf{0 . 8 5}$ & $\mathbf{2 . 4 7}$ & $\mathbf{0 . 7 4}$ & -0.067 & $\mathbf{0 . 0 6}$ & $\mathbf{0 . 2 1}$ \\
\hline (Formica et al., 2012) & 1.19 & 1.67 & 0.21 & -0.178 & -- & -- \\
\hline (Leger and Milner, 2000) & 2.2 & -- & -- & -- & -- & -- \\
\hline $\begin{array}{l}\text { (De Serres and Milner, } \\
\text { 1991) }\end{array}$ & 3 & -- & -- & -- & -- & - \\
\hline (van Eesbeek et al., 2010) & 3.05 & -- & -- & -- & -- & -- \\
\hline
\end{tabular}

We observed the short-range-stiffness (SRS) phenomenon within the first $2^{\circ}$ of each movement (the data from this region was excluded from the final, linear stiffness matrix). Gender and direction of movement (outbound vs. inbound) were statistically significant factors in ellipsoid volume, with outbound volume being higher than inbound volume and male volume being higher then female volume. Torque-position plots exhibited hysteresis; this effect is responsible for the dependence of ellipsoid volume on direction of movement. Repetition of movement did not have a statistically significant effect on any of our measures of passive stiffness (ratio of eigenvectors, orientation of eigenvectors, volume of stiffness ellipsoid, etc). This finding indicates that thixotropy, while present in the joint's viscoelastic behavior, does not affect wrist stiffness. It was found that the major eigenvector aligned with the RUD axis of rotation and was roughly five times as large as the intermediate and minor axes, which were not statistically aligned with FE or PS. However, because the stiffness ellipse in the FE-PS plane is 
roughly circular in form, this finding indicates that movements in RUD require five times as much torque as movements of identical ranges in FE or PS.

Our findings are consistent with those of previous studies. Our finding of SRS within the first $2^{\circ}$ is similar to Axelson and Hagbarth's report of SRS within the first $3^{\circ}-4^{\circ}$ of movement (Axelson and Hagbarth, 2001). The calculated average stiffness of $0.73-0.96 \mathrm{Nm} / \mathrm{rad}$ in FE and 2.16-2.78 Nm/rad in RUD (female-male) fall solidly in the mid range of reported stiffness values and are consistent with the most recent characterizations of non-cadaveric, quasi-static passive stiffness estimates.

In addition to these similarities, important differences were found. The difference between the RUD axis and the major eigenvector (in the FE-RUD plane) for our study was of $2.2 \pm 4.1^{\circ}$ as compared to the $21 \pm 9.2^{\circ}$ difference reported by Formica (Formica et al., 2012). Potential origins of this difference include 1) the difference in neutral position defined in each study, and 2) the possibility that averaging the coupling effect across the $\pm 15^{\circ} \mathrm{ROM}$ in PS studied in our 3DOF work results in an apparent reduction of coupling, when in fact the coupling in 2 DOF remains the same. Also, the average stiffness of $0.73-0.75 \mathrm{Nm} / \mathrm{rad}$ in PS (female-male) was significantly higher than that reported by Formica, which was $0.12-0.25 \mathrm{Nm} / \mathrm{rad}$ (femalemale) (Formica et al., 2012).

Future studies should make improvements based on our findings and extend the envelope of knowledge with deeper investigations into the factors that, of necessity, we had to fix as constant. While our study provides a thorough of the $\mathrm{ROM} \pm 15^{\circ}$, this range only encompasses $40 \%$ of the functional ROM (f-ROM) in FE and 70\% of the f-ROM in RUD (percentages based on an average of the f-ROM values presented in Chapter 2). New studies should endeavor to characterize passive stiffness throughout the full f-ROM used by the neuromuscular system to 
accomplish daily tasks. Eventually, the entire extreme ROM should be characterized. Of necessity, we held constant the movement velocity used in our study; further tests should be performed to find the relationship between movement velocity and passive stiffness. Using the results of Gehrmann et al.'s circumduction study (Gehrmann et al., 2008), we have extrapolated a correlation between finger constraint and passive stiffness. Such a correlation, if it can be demonstrated and quantified, may have a significant effect on joint torques during occupational tasks that involve grasping a handle. To date, no studies have investigated the effect of handedness on joint stiffness. Lastly, additional characterization of phenomena such as hysteresis, stress relaxation, and thixotropy is needed.

Taking the current research and all previous findings as our foundation, the area of study circumscribed by passive wrist stiffness has a strong knowledge base with ample room for additional study. The results of the present study are sufficient to allow for computer models of the arm to include the coupled stiffness of the wrist and forearm for the first time. It is anticipated that such models will now be better suited to approach an answer to the question "Why does the neuromuscular system move the wrist and forearm the way it does?" and to investigate the possible correlation between "paths of least resistance" in the forearm-wrist joint space with the orientations assumed by the elbow and shoulder joints during activities of daily living. 


\section{REFERENCES}

AAOS, 1965. Joint Motion: Methods of Measuring and Recording. AAOS, Chicago.

Adams, B.D., Grosland, N.M., Murphy, D.M., McCullough, M., 2003. Impact of impaired wrist motion on hand and upper-extremity performance. Journal of Hand Surgery-American Volume 28A, 898-903.

Aizawa, J., Masuda, T., Koyama, T., Nakamaru, K., Isozaki, K., Okawa, A., Morita, S., 2010. Three-dimensional motion of the upper extremity joints during various activities of daily living. $J$ Biomech 43, 2915-2922.

Andrews, J.G., Youm, Y., 1979. Biomechanical Investigation of Wrist Kinematics. J Biomech 12, 83-93.

Axelson, H.W., Hagbarth, K.E., 2001. Human motor control consequences of thixotropic changes in muscular short-range stiffness. Journal of Physiology-London 535, 279-288.

Boone, D.C., Azen, S.P., 1979. Normal Range of Motion of Joints in Male-Subjects. Journal of Bone and Joint Surgery-American Volume 61, 756-759.

Brumbaugh, R.B., Crowninshield, R.D., Blair, W.F., Andrews, J.G., 1982. An Invivo Study of Normal Wrist Kinematics. Journal of Biomechanical Engineering-Transactions of the Asme 104, 176-181.

Brumfield, R.H., Champoux, J.A., 1984. A biomechanical study of normal functional wrist motion. Clin Orthop Relat Res, 23-25.

Brumfield, R.H., Jr., Nickel, V.L., Nickel, E., 1966. Joint motion in wrist flexion and extension. Southern medical journal 59, 909-910.

Bulthaup, S., Cipriani, D.J., Thomas, J.J., 1999. An electromyography study of wrist extension orthoses and upper-extremity function. American Journal of Occupational Therapy 53, 434-440.

Campbell, K.S., 2010. Short-range mechanical properties of skeletal and cardiac muscles. Advances in experimental medicine and biology 682, 223-246. 
Carlson, J.D., Trombly, C.A., 1983. THE EFFECT OF WRIST IMMOBILIZATION ON PERFORMANCE OF THE JEBSEN HAND FUNCTION-TEST. American Journal of Occupational Therapy 37, 167-175.

Chan, W.Y.Y., Chapparo, C., 1999. Effect of wrist immobilisation on upper limb function of elderly males. Technology and Disability 11, 39-49.

Charles, S.K., Hogan, N., 2010. The curvature and variability of wrist and arm movements. Exp Brain Res 203, 63-73.

Charles, S.K., Hogan, N., 2011. Dynamics of wrist rotations. J Biomech 44, 614-621.

Charles, S.K., Hogan, N., 2012. Stiffness, not inertial coupling, determines path curvature of wrist motions. J Neurophysiol 107, 1230-1240.

Crisco, J.J., Heard, W.M.R., Rich, R.R., Paller, D.J., Wolfe, S.W., 2011. The mechanical axes of the wrist are oriented obliquely to the anatomical axes. The Journal of Bone \& Joint Surgery 93, 169-177.

de Leva, P., 1996. Adjustments to Zatsiorsky-Seluyanov's segment inertia parameters. J Biomech $29,1223-1230$.

de Rugy, A., Davoodi, R., Carroll, T.J., 2012. Changes in wrist muscle activity with forearm posture: implications for the study of sensorimotor transformations. J Neurophysiol 108, 28842895.

De Serres, S.J., Milner, T.E., 1991. Wrist muscle activation patterns and stiffness associated with stable and unstable mechanical loads. Exp Brain Res 86, 451-458.

Drake, W.B., Charles, S.K., Passive stiffness of coupled wrist and forearm rotations. In review.

Foley, M., Silverstein, B., Polissar, N., 2007. The economic burden of carpal tunnel syndrome: long-term earnings of CTS claimants in Washington State. Am J Ind Med 50, 155-172.

Formica, D., Charles, S.K., Zollo, L., Guglielmelli, E., Hogan, N., Krebs, H.I., 2012. The Passive Stiffness of the Wrist and Forearm. J Neurophysiol 108, 1158-1166.

Gehrmann, S.V., Kaufmann, R.A., Li, Z.M., 2008. Wrist Circumduction Reduced by Finger Constraints. Journal of Hand Surgery-American Volume 33A, 1287-1292.

Gielen, C., Houk, J.C., 1984. Nonlinear Viscosity of Human Wrist. J Neurophysiol 52, 553-569.

Gillard, D.M., Yakovenko, S., Cameron, T., Prochazka, A., 2000. Isometric muscle lengthtension curves do not predict angle-torque curves of human wrist in continuous active movements. J Biomech 33, 1341-1348. 
Goto, A., Moritomo, H., Murase, T., Oka, K., Sugamoto, K., Arimura, T., Masumoto, J., Tamura, S., Yoshikawa, H., Ochi, T., 2005. In vivo three-dimensional wrist motion analysis using magnetic resonance imaging and volume-based registration. Journal of Orthopaedic Research 23, 750-756.

Gunal, I., Kose, N., Erdogan, O., Gokturk, E., Seber, S., 1996. Normal range of motion of the joints of the upper extremity in male subjects, with special reference to side. The Journal of bone and joint surgery. American volume 78, 1401-1404.

Halaki, M., O'Dwyer, N., Cathers, I., 2006. Systematic nonlinear relations between displacement amplitude and joint mechanics at the human wrist. J Biomech 39, 2171-2182.

Hill, D., 1968. Tension due to interaction between the sliding filaments in resting striated muscle. The effect of stimulation. The Journal of physiology 199, 637-684.

Hogan, N., 1985. The Mechanics of Multi-Joint Posture and Movement Control. Biological Cybernetics 52, 315-331.

Horii, E., An, K.N., Linscheid, R.L., 1993. Excursion of prime wrist tendons. J Hand Surg Am $18,83-90$.

Jim Albers, S.H., Yong-Ku Kong, 2003. NIOSH Health Hazard Evaluation Report: Genesis Steel Services. HHE Report.

Johns, R.J., Wright, V., 1962. Relative Importance of Various Tissues in Joint Stiffness. Journal of Applied Physiology 17, 824-828.

Kaufmann, R., Pfaeffle, J., Blankenhorn, B., Stabile, K., Robertson, D., Goitz, R., 2005. Kinematics of the midcarpal and radiocarpal joints in radioulnar deviation: An in vitro study. Journal of Hand Surgery-American Volume 30A, 937-942.

Kaufmann, R.A., Pfaeffle, H.J., Blankenhorn, B.D., Stabile, K., Robertson, D., Goitz, R., 2006. Kinematics of the midcarpal and radiocarpal joint in flexion and extension: An in vitro study. Journal of Hand Surgery-American Volume 31A, 1142-1148.

King, S., Thomas, J.J., Rice, M.S., 2003. The immediate and short-term effects of a wrist extension orthosis on upper-extremity kinematics and range of shoulder motion. American Journal of Occupational Therapy 57, 517-524.

Kobayashi, M., Berger, R.A., Linscheid, R.L., An, K.N., 1997. Intercarpal kinematics during wrist motion. Hand Clin 13, 143-149.

Krebs, H.I., Volpe, B.T., Williams, D., Celestino, J., Charles, S.K., Lynch, D., Hogan, N., 2007. Robot-aided neurorehabilitation: A robot for wrist rehabilitation. Ieee T Neur Sys Reh 15, 327335. 
Leger, A.B., Milner, T.E., 2000. Passive and active wrist joint stiffness following eccentric exercise. European Journal of Applied Physiology 82, 472-479.

Lehman, S.L., Calhoun, B.M., 1990. An identified model for human wrist movements. Exp Brain Res 81, 199-208.

Leonard, L., Sirkett, D., Mullineux, G., Giddins, G.E.B., Miles, A.W., 2005. Development of an in-vivo method of wrist joint motion analysis. Clinical Biomechanics 20, 166-171.

Li, Z.M., 2002. The influence of wrist position on individual finger forces during forceful grip. $\mathrm{J}$ Hand Surg Am 27, 886-896.

Li, Z.M., Kuxhaus, L., Fisk, J.A., Christophel, T.H., 2005. Coupling between wrist flexionextension and radial-ulnar deviation. Clinical Biomechanics 20, 177-183.

Marshall, M.M., Mozrall, J.R., Shealy, J.E., 1999. The effects of complex wrist and forearm posture on wrist range of motion. Hum Factors 41, 205-213.

May-Lisowski, T.L., King, P.M., 2008. Effect of wearing a static wrist orthosis on shoulder movement during feeding. American Journal of Occupational Therapy 62, 438-445.

Mell, A.G., Childress, B.L., Hughes, R.E., 2005. The effect of wearing a wrist splint on shoulder kinematics during object manipulation. Arch Phys Med Rehab 86, 1661-1664.

Mell, A.G., Friedman, M.A., Hughes, R.E., Carpenter, J.E., 2006. Shoulder muscle activity increases with wrist splint use during a simulated upper-extremity work task. American Journal of Occupational Therapy 60, 320-326.

Milner, T.E., Cloutier, C., 1993. Compensation for mechanically unstable loading in voluntary wrist movement. Exp Brain Res 94, 522-532.

Milner, T.E., Cloutier, C., 1998. Damping of the wrist joint during voluntary movement. Exp Brain Res 122, 309-317.

Mussa-Ivaldi, F.A., Hogan, N., Bizzi, E., 1985. Neural, Mechanical, and Geometric Factors Subserving Arm Posture in Humans. J Neurosci 5, 2732-2743.

Nelson, D.L., 1997. Functional wrist motion. Hand Clinics 13, 83-\&.

Neu, C.P., Crisco, J.J., Wolfe, S.W., 2001. In vivo kinematic behavior of the radio-capitate joint during wrist flexion-extension and radio-ulnar deviation. J Biomech 34, 1429-1438.

Ochi, E., Nakazato, K., Ishii, N., 2007. Effects of eccentric exercise on joint stiffness and muscle connectin (titin) isoform in the rat hindlimb. The journal of physiological sciences : JPS 57, 1-6. 
Ojima, H., Miyake, S., Kumashiro, M., Togami, H., Suzuki, K., 1992. Ranges of dynamic motion of the wrist in healthy young and middle-aged men. Ergonomics 35, 1467-1477.

Pagnotta, A., Korner-Bitensky, N., Mazer, B., Baron, M., Wood-Dauphinee, S., 2005. Static wrist splint use in the performance of daily activities by individuals with rheumatoid arthritis. Journal of Rheumatology 32, 2136-2143.

Palmer, A.K., Werner, F.W., Murphy, D., Glisson, R., 1985. Functional wrist motion - A biomechanical study. Journal of Hand Surgery-American Volume 10A, 39-46.

Pando, A., Lee, H., Drake, W.B., Hogan, N., Charles, S.K., Position-dependent characterization of passive wrist stiffness. In review.

Patterson, R.M., Nicodemus, C.L., Viegas, S.F., Elder, K.W., Rosenblatt, J., 1998. High-speed, three-dimensional kinematic analysis of the normal wrist. Journal of Hand Surgery-American Volume 23A, 446-453.

Proske, U., Morgan, D.L., Gregory, J.E., 1993. Thixotropy in skeletal muscle and in muscle spindles: a review. Progress in neurobiology 41, 705-721.

Rack, P.M., Westbury, D.R., 1974. The short range stiffness of active mammalian muscle and its effect on mechanical properties. The Journal of physiology 240, 331-350.

Rijnveld, N., Krebs, H., Year Passive Wrist Joint Impedance in Flexion-Extension and Abduction-Adduction. In International Conference on Rehabilitation Robotics. Netherlands.

Ritt, M.J., Stuart, P.R., Berglund, L.J., Berger, R.A., Linscheid, R.L., Cooney, W.P., 3rd, An, K.N., 1996. Rotational laxity and stiffness of the radiocarpal joint. Clin Biomech (Bristol, Avon) $11,227-232$.

Ryu, J., Cooney, W.P., Askew, L.J., An, K.N., Chao, E.Y.S., 1991a. Functional ranges of motion of the wrist joint. Journal of Hand Surgery-American Volume 16A, 409-419.

Ryu, J., Palmer, A.K., Cooney, W.P., 1991b. Wrist Joint Motion, in: An, K.N., Berger, R.A., Cooney, W.P. (Eds.), Biomechanics of the Wrist Joint. Springer Verlag, New York, pp. 48-55.

Salvia, P., Woestyn, L., David, J.H., Feipel, V., Van, S., Jan, S., Klein, P., Rooze, M., 2000. Analysis of helical axes, pivot and envelope in active wrist circumduction. Clinical Biomechanics 15, 103-111.

Sarrafian, S.K., Melamed, J.L., Goshgarian, G.M., 1977. Study of wrist motion in flexion and extension. Clin Orthop Relat Res, 153-159.

Shu, Y., Mirka, G.A., 2006. A laboratory study of the effects of wrist splint orthoses on forearm muscle activity and upper extremity posture. Human Factors 48, 499-510. 
Sommer, H.J., Miller, N.R., 1980. A Technique for Kinematic Modeling of Anatomical Joints. Journal of Biomechanical Engineering-Transactions of the Asme 102, 311-317.

Valero-Cuevas, F.J., Small, C.F., 1997. Load dependence in carpal kinematics during wrist flexion in vivo. Clinical Biomechanics 12, 154-159.

van Andel, C.J., Wolterbeek, N., Doorenbosch, C.A.M., Veeger, D., Harlaar, J., 2008. Complete 3D kinematics of upper extremity functional tasks. Gait \& Posture 27, 120-127.

van Eesbeek, S., de Groot, J.H., van der Helm, F.C., de Vlugt, E., 2010. In vivo estimation of the short-range stiffness of cross-bridges from joint rotation. J Biomech 43, 2539-2547.

Wagner, C.H., 1988. The pianist's hand: anthropometry and biomechanics. Ergonomics 31, 97131.

Whitehead, N.P., Morgan, D.L., Gregory, J.E., Proske, U., 2003. Rises in whole muscle passive tension of mammalian muscle after eccentric contractions at different lengths. J Appl Physiol 95, 1224-1234.

Whitehead, N.P., Weerakkody, N.S., Gregory, J.E., Morgan, D.L., Proske, U., 2001. Changes in passive tension of muscle in humans and animals after eccentric exercise. The Journal of physiology 533, 593-604.

Wu, G., van der Helm, F.C.T., Veeger, H.E.J., Makhsous, M., Van Roy, P., Anglin, C., Nagels, J., Karduna, A.R., McQuade, K., Wang, X.G., Werner, F.W., Buchholz, B., 2005. ISB recommendation on definitions of joint coordinate systems of various joints for the reporting of human joint motion - Part II: shoulder, elbow, wrist and hand. J Biomech 38, 981-992.

Yoo, I.G., Jung, M.Y., Jeon, H.S., Lee, J., 2010. Effects of Wrist-extension Orthosis on Shoulder and Scapular Muscle Activities during Simulated Assembly Tasks. Industrial Health 48, 108114.

Youm, Y., McMurthy, R.Y., Flatt, A.E., Gillespie, T.E., 1978. Kinematics of the wrist. I. An experimental study of radial-ulnar deviation and flexion-extension. The Journal of bone and joint surgery. American volume 60, 423-431. 